The Astrophysical Journal, 502:315-336, 1998 July 20

(C) 1998. The American Astronomical Society. All rights reserved. Printed in U.S.A.

\title{
ENVELOPE STRUCTURE ON 700 AU SCALES AND THE MOLECULAR OUTFLOWS OF LOW-MASS YOUNG STELLAR OBJECTS
}

\author{
MichIEL R. HogerheIJDe AND EWINe F. VAN DishoeCK \\ Sterrewacht Leiden, P.O. Box 9513, 2300 RA, Leiden, The Netherlands \\ GEOFFREY A. BLAKE \\ Division of Geological and Planetary Sciences, California Institute of Technology, MS 150-21, Pasadena, CA 91125 \\ AND \\ HuIB JAN VAN LANGEVELDE \\ Joint Institute for VLBI in Europe, P.O. Box 2, 7990 AA, Dwingeloo, The Netherlands \\ Received 1997 September 11; accepted 1998 February 26
}

\begin{abstract}
Aperture synthesis observations of $\mathrm{HCO}^{+} J=1-0,{ }^{13} \mathrm{CO} 1-0$, and $\mathrm{C}^{18} \mathrm{O} 1-0$ obtained with the Owens Valley Millimeter Array are used to probe the small-scale $\left(5^{\prime \prime} \approx 700 \mathrm{AU}\right)$ structure of the molecular envelopes of a well-defined sample of nine embedded low-mass young stellar objects in Taurus. The interferometer results can be understood in terms of: (1) a core of radius $\$ 1000$ AU surrounding the central star, possibly flattened and rotating; (2) condensations scattered throughout the envelope that may be left over from the inhomogeneous structure of the original cloud core or that may have grown during collapse; and (3) material within the outflow or along the walls of the outflow cavity. Masses of the central cores are $0.001-0.1 M_{\odot}$, and agree well with dust continuum measurements. Averaged over the central $20^{\prime \prime}(3000 \mathrm{AU})$ region, an $\mathrm{HCO}^{+}$abundance of $4 \times 10^{-8}$ is inferred, with a spread of a factor of 3 between the different sources. Reanalysis of previously presented single-dish data yields an $\mathrm{HCO}^{+}$ abundance of $(5.0 \pm 1.7) \times 10^{-9}$, which may indicate an average increase by a factor of a few on the smaller scales sampled by the interferometer. Part of this apparent abundance variation could be explained by contributions from extended cloud emission to the single-dish $\mathrm{C}^{18} \mathrm{O}$ lines, and uncertainties in the assumed excitation temperatures and opacities. The properties of the molecular envelopes and outflows are further investigated through single-dish observations of ${ }^{12} \mathrm{CO} J=6-5,4-3$, and $3-2,{ }^{13} \mathrm{CO}$ 6-5 and 3-2, and $\mathrm{C}^{18} \mathrm{O} 3-2$ and 2-1, obtained with the James Clerk Maxwell and IRAM $30 \mathrm{~m}$ telescopes, along with the Caltech Submillimeter Observatory. Ratios of the mid- $J$ CO lines are used to estimate the excitation temperature, with values of $25-80 \mathrm{~K}$ derived for the gas near line center. The outflow wings show a similar range, although $T_{\mathrm{ex}}$ is enhanced by a factor of 2-3 in at least two sources. In contrast to the well-studied L1551 IRS 5 outflow, which extends over $10^{\prime}(0.4 \mathrm{pc})$, seven of the remaining eight sources are found to drive ${ }^{12} \mathrm{CO} 3-2$ outflows over $\leq 1^{\prime}(0.04 \mathrm{pc})$; only L1527 IRS has a well-developed outflow of some $3^{\prime}(0.12 \mathrm{pc})$. Estimates are obtained for the outflow kinetic luminosity, $L_{\mathrm{kin}}$, and the flow momentum rate, $F_{\mathrm{CO}}$, applying corrections for line opacity and source inclination. The flow force $F_{\text {CO }}$ correlates with the envelope mass and with the $2.7 \mathrm{~mm}$ flux of the circumstellar disk. Only a weak correlation is seen with $L_{\mathrm{bol}}$, while none is found with the relative age of the object as measured by $\int T_{\mathrm{mb}}\left(\mathrm{HCO}^{+} 3-2\right) d V / L_{\mathrm{bol}}$. These trends support the hypothesis that outflows are driven by accretion through a disk, with a global mass infall rate determined by the mass and density of the envelope. The association of compact $\mathrm{HCO}^{+}$emission with the walls of the outflow cavities indicates that outflows in turn influence the appearance of the envelopes. It is not yet clear, however, whether they are actively involved in sweeping up envelope material, or merely provide a low-opacity pathway for heating radiation to reach into the envelope.
\end{abstract}

Subject headings: ISM: molecules — radio lines: stars - stars: formation -

stars: low mass, brown dwarfs - stars: pre-main-sequence

\section{INTRODUCTION}

In the earliest stages of their formation, low-mass young stellar objects (YSOs) are embedded in an envelope of gas and dust several thousand AU in radius, and are often surrounded by a $<100$ AU disk (see Shu et al. 1993 for an overview of star formation). Theoretical models of cloud core collapse predict a density structure characterized by a radial power law (e.g., Terebey, Shu, \& Cassen 1984; Galli \& Shu 1993; Fiedler \& Mouschovias 1992, 1993; Boss 1993). The envelopes may show an inwardly increasing degree of flattening, as well as rotation or infall. Many YSOs drive a bipolar outflow, which may play a pivotal role in their evolution. Outflows are thought to be powered by the interaction of a magnetic field with a rotating accretion disk (see Königl \& Ruden 1993; Bachiller 1996), and carry away angular momentum that would otherwise prevent accretion. By sweeping up envelope material, they may be involved in reversing infall and clearing the envelope around the YSO (Raga \& Cabrit 1993; Li \& Shu 1996; Padman, Bence, \& Richer 1997). Cabrit \& Bertout (1990, 1992), Cabrit \& André (1991), Bontemps et al. (1996), and Saraceno et al. (1996) have investigated the relation between outflow kinetic luminosity, bolometric luminosity, and envelope continuum flux. They conclude that stronger 
outflows are associated with sources that have higher luminosities and more massive envelopes.

Several important questions are raised by these findings. For example, how does the structure of the envelope on 1000 AU scales compare to theoretical predictions? To what extent do the outflows impact the structure and appearance of the envelopes? What is the relation between the outflow and source properties like stellar mass, evolutionary state, luminosity, envelope mass, and the presence of a circumstellar disk? In this paper, the small-scale $\left(5^{\prime \prime} \approx 700 \mathrm{AU}\right)$ structure of the molecular envelopes around a sample of nine embedded, low-mass YSOs in Taurus (Table 1) is investigated. These objects are probably in the stage at which the outflow is terminating infall and clearing away the envelopes. They were selected on the basis of their single-dish $\mathrm{HCO}^{+} 3-2$ emission from the IRAS flux- and color-limited sample of YSOs in Taurus-Auriga $(d=140$ pc) defined by Tamura et al. (1991). The bolometric luminosities of the sources range between 0.66 and $25.5 L_{\odot}$, with corresponding upper limits to their stellar mass of $0.15-2.7$ $M_{\odot}$, assuming that all luminosity is stellar and that the objects are located on the birth line (Stahler 1988; Palla \& Stahler 1993).

In a previous paper (Hogerheijde et al. 1997a, hereafter Paper I) these sources were studied through interferometry of the 3.4 and $2.7 \mathrm{~mm}$ continuum emission, $1.1 \mathrm{~mm}$ singledish continuum observations from the literature, and $\mathrm{HCO}^{+}$and $\mathrm{H}^{13} \mathrm{CO}^{+} J=1-0,3-2$, and 4-3 single-dish line observations. We found that at least two-thirds of the sources are surrounded by compact $\left(<3^{\prime \prime}\right)$ disks with 2.7 mm fluxes of 6-100 mJy and masses of $0.005-0.07 M_{\odot}$, assuming optically thin emission. Between $30 \%$ and $75 \%$ of the $1.1 \mathrm{~mm}$ single-dish flux observed in a 19" beam could be attributed to these disks, the remainder tracing envelopes of 0.001-0.26 $\mathrm{M}_{\odot}$. The $\mathrm{HCO}^{+}$emission was well correlated with the $1.1 \mathrm{~mm}$ envelope flux, and both could be described simultaneously with the simple inside-out collapse model of Shu (1977) or closely related power-law models with slopes between 1 and 3. A beam-averaged $\mathrm{HCO}^{+} / \mathrm{H}_{2}$ abundance of $(1.2 \pm 0.4) \times 10^{-8}$ was inferred from optically thin single-dish $\mathrm{H}^{13} \mathrm{CO}^{+} 1-0$ and $\mathrm{C}^{18} \mathrm{O} 1-0$ lines (Mizuno et al. 1994; Hayashi et al. 1994), not including corrections for the beam efficiency of the Nobeyama $45 \mathrm{~m}$ telescope. In the present paper, we use beam efficiencies of 0.8 at $\mathrm{H}^{13} \mathrm{CO}^{+}$ 1-0 and 0.4 at $\mathrm{C}^{18} \mathrm{O} 1-0$ (cf. Kitamura et al. 1990; Hayashi et al. 1994), resulting in a lower value for the single-dish $\mathrm{HCO}^{+}$abundance of $(5.0 \pm 1.7) \times 10^{-9}$. Paper I showed that $\mathrm{HCO}^{+}$, especially in its 3-2 and 4-3 lines, is an excellent tracer of the envelopes. This led us to propose the ratio $\int T_{\mathrm{mb}}\left(\mathrm{HCO}^{+} 3-2\right) d V / L_{\mathrm{bol}}$ as an evolutionary tracer for the embedded phase, as it corresponds to the current ratio of envelope mass to stellar mass.

Over the past decade, millimeter interferometry has developed into a powerful tool for the study of star formation through the increase in the sensitivity and number of elements of the various arrays, making possible a detailed investigation of a reasonably extended sample of YSOs via their millimeter line emission. Observations of our objects at the Owens Valley Millimeter Array have resulted in highquality $\mathrm{HCO}^{+} 1-0,{ }^{13} \mathrm{CO} 1-0$, and $\mathrm{C}^{18} \mathrm{O} 1-0$ data with $3^{\prime \prime}-5^{\prime \prime}$ resolution, tracing the inner few thousand AU of the protostellar envelopes. Previous interferometric studies toward some of the sources have been undertaken in isotopic lines of CO by Sargent et al. (1988), Terebey, Vogel, \& Myers (1989), Terebey et al. (1990), Chandler et al. (1996), Momose et al. (1996), Zhou, Evans, \& Wang (1996), Tamura et al. (1996), and Ohashi et al. (1997a, 1997b); in $\mathrm{HCO}^{+}$and $\mathrm{H}^{13} \mathrm{CO}^{+}$by Rudolph (1992), van Langevelde, van Dishoeck, \& Blake (1994a), and Saito et al. (1996); and in CS by Ohashi et al. (1996b). The study presented here is one of the first combining interferometry data of a well-defined sample of YSOs with $10^{\prime \prime}-30^{\prime \prime}$ resolution single-dish observations of ${ }^{12} \mathrm{CO} 6-5,4-3$, and $3-2,{ }^{13} \mathrm{CO} 6-5$ and $3-2$, and $\mathrm{C}^{18} \mathrm{O} 3-2$ and $2-1$, thereby covering a large range of physical conditions and spatial scales.

The widespread detection of ${ }^{12} \mathrm{CO}$ and ${ }^{13} \mathrm{CO} 6-5$ toward our sample, which traces gas of $\sim 80 \mathrm{~K}$, led Spaans et al. (1995) to propose a model in which scattered ultraviolet radiation from the star-disk boundary layer is responsible for heating the surroundings of the outflow cavity. ${ }^{12} \mathrm{CO}$ 3-2 maps of the outflows are used here to investigate their relationship to the structure seen in the interferometer maps. All our sources are associated with outflow emission (cf. Snell, Loren, \& Plambeck 1980; Edwards \& Snell 1982;

TABLE 1

SOURCE SAMPLE

\begin{tabular}{|c|c|c|c|c|c|c|c|c|}
\hline Source & $I R A S$ PSC & $\alpha(1950.0)$ & $\delta(1950.0)$ & $\begin{array}{c}\text { Visible/ } \\
\text { Embedded }\end{array}$ & $\begin{array}{c}L_{\mathrm{bol}} \\
\left(L_{\odot}\right)\end{array}$ & $\begin{array}{c}M_{*}^{\mathrm{a}} \\
\left(M_{\odot}\right)\end{array}$ & $\begin{array}{c}M_{\text {disk }}^{\mathrm{b}} \\
\left(M_{\odot}\right)\end{array}$ & $\begin{array}{l}M_{\text {env }}^{\mathrm{c}} \\
\left(M_{\odot}\right)\end{array}$ \\
\hline L1489 IRS & $04016+2610$ & 040140.5 & +261048 & Embedded & 3.70 & 0.4 & $\leq 0.004$ & $0.016-0.025$ \\
\hline T Tau ....... & $04190+1924$ & 041904.1 & +192506 & Visible $^{\mathrm{d}}$ & $25.50^{\mathrm{e}}$ & $2.7^{\mathrm{e}}$ & 0.023 & 0.029 \\
\hline Haro 6-10 & $04263+2426$ & 042621.9 & +242629 & Visible $^{d}$ & 6.98 & 0.9 & 0.010 & $<0.004$ \\
\hline L1551 IRS $5 \ldots$ & $04287+1801$ & 042840.2 & +180142 & Embedded & 21.90 & 2.6 & 0.073 & 0.26 \\
\hline L1535 IRS. & $04325+2402$ & 043233.4 & +240213 & Embedded & 0.70 & 0.15 & $\leq 0.005$ & $<0.010$ \\
\hline TMR $1 \ldots \ldots$ & $04361+2547$ & 043609.7 & +254729 & Embedded & 2.90 & 0.3 & 0.009 & 0.007 \\
\hline TMC 1A.. & $04365+2535$ & 043631.1 & +253554 & Embedded & 2.20 & 0.3 & 0.020 & 0.018 \\
\hline L1527 IRS . & $04368+2557$ & 043649.6 & +255721 & Embedded & 1.30 & 0.2 & 0.017 & 0.031 \\
\hline TMC $1 \ldots$ & $04381+2540$ & 043808.4 & +254052 & Embedded & 0.66 & 0.15 & $\leq 0.005$ & $0.005-0.016$ \\
\hline
\end{tabular}

NoTE.-Units of right ascension are hours, minutes, and seconds, and units of declination are degrees, arcminutes, and arcseconds.

${ }^{a}$ Maximum mass of central object, assuming that all luminosity is stellar and that the object is on the birth line.

${ }^{\mathrm{b}}$ Mass of circumstellar disk inferred from 3.4 and $2.7 \mathrm{~mm}$ interferometer observations, assuming an average dust temperature of 30 $\mathrm{K}$ and optically thin radiation (Paper I).

' Envelope mass within a 19" beam, assuming an average dust temperature of $30 \mathrm{~K}$ (Paper I).

${ }^{\mathrm{d}}$ With embedded companion.

e Sum of T Tau N and S.

REFERENCES.-For $L_{\mathrm{bol}}$, T Tau, Cohen, Emerson, \& Beichman 1989; for $L_{\mathrm{bol}}$, Kenyon \& Hartmann 1995; for positions, $M_{*}, M_{\mathrm{disk}}$, and $M_{\text {env }}$, Paper I. 
Frerking \& Langer 1982; Terebey et al. 1989; MoriartySchieven et al. 1992), but only a few had been mapped previously with a resolution of $10^{\prime \prime}-15^{\prime \prime}$, comparable to the interferometer maps (T Tau by Schuster, Harris, \& Russell 1997; L1551 IRS 5 by Moriarty-Schieven \& Snell 1988; TMC 1A and TMC 1 by Chandler et al. 1996; L1527 IRS by MacLeod et al. 1994).

In this paper, the structure of the molecular envelopes on small scales (700 AU) around low-mass YSOs is studied through interferometric observations of $\mathrm{HCO}^{+},{ }^{13} \mathrm{CO}$, and $\mathrm{C}^{18} \mathrm{O}$, combined with high-resolution single-dish data for these species. In particular, the influence of the bipolar outflow on this structure will be investigated. Outflow properties will be derived and compared to intrinsic source characteristics like envelope mass, luminosity, and disk continuum flux. The aim of this paper is to arrive at conclusions about the envelope structure of our sample of YSOs as a whole, which sometimes requires the use of simplifying assumptions. The outline of the paper is as follows. In $\S 2$ the details of the observations are presented. The results of the millimeter interferometry are discussed in $\S 3$, where estimates are inferred for the mass of the probed material and the $\mathrm{HCO}^{+}$abundance, and compared to single-dish results from Paper I. In $\S 4$ the single-dish results are presented. Constraints are derived for the molecular excitation (§ 4.1) and the properties of the outflows (§ 4.2). In $\S 4.3$ the relation between outflow strength, envelope mass, and disk flux is examined. The envelope structure of the individual sources is discussed in detail in $\S 5$, and the main conclusions are summarized in $\S 6$.

\section{OBSERVATIONS}

The sources of our sample are listed in Table 1; an overview of the data presented in this paper is given in Table 2 . The following subsections discuss the details of the observations.

\subsection{Millimeter Interferometer Observations}

Observations of $\mathrm{HCO}^{+} 1-0(89.188523 \mathrm{GHz}),{ }^{13} \mathrm{CO} 1-0$ $(110.201370 \mathrm{GHz})$, and $\mathrm{C}^{18} \mathrm{O} 1-0(109.782182 \mathrm{GHz})$ were obtained with the Owens Valley Radio Observatory (OVRO) Millimeter Array ${ }^{1}$ between 1992 and 1997, simultaneously with the 3.4 and $2.7 \mathrm{~mm}$ continuum emission presented in Paper I. During the $89 \mathrm{GHz}$ observations, the array consisted of five antennas; the $110 \mathrm{GHz}$ observations were made with a six element array. Data taken in the low-resolution and equatorial configurations were combined, resulting in a $u-v$ coverage with spacing between 4 and $40 \mathrm{k} \lambda$ at $89 \mathrm{GHz}$ and between 4 and $80 \mathrm{k} \lambda$ at $110 \mathrm{GHz}$. This corresponds to naturally weighted, synthesized beams of $5^{\prime \prime}$ and $3^{\prime \prime}$ FWHM, respectively. The observations of T Tau were made in five different array configurations, and have been presented by van Langevelde et al. (1994a). Spectral line data were recorded in two 64 channel bands with respective widths of 2 and $8 \mathrm{MHz}$, resulting in velocity resolutions of 0.11 and $0.42 \mathrm{~km} \mathrm{~s}^{-1}$ at $89 \mathrm{GHz}$, and 0.09 and $0.34 \mathrm{~km} \mathrm{~s}^{-1}$ at $110 \mathrm{GHz}$. The observations of $\mathrm{T}$ Tau in ${ }^{13} \mathrm{CO} 1-0$ were obtained with a lower resolution of $0.68 \mathrm{~km}$ $\mathrm{s}^{-1}$. Visibility data were calibrated using the MMA package, developed specifically for OVRO (Scoville et al. 1993). The quasars PKS $0333+321$ and $0528+134$ served

\footnotetext{
${ }^{1}$ The Owens Valley Millimeter Array is operated by the California Institute of Technology under funding from the U.S. National Science Foundation (AST96-13717).
}

as phase calibrators $(0420-014$ for the observations of $\mathrm{T}$ Tau); the amplitudes were calibrated on $3 \mathrm{C} 454.3$ and $3 \mathrm{C}$ 273 , whose fluxes at the time were determined from observations of the planets. Calibration of the correlator passbands used noise integrations and observations of 3C 454.3 and $3 \mathrm{C} 273$. The quasar $0528+134$ cannot be used for passband calibration of $\mathrm{HCO}^{+}$observations of sources in the Taurus region because of strong Galactic $\mathrm{HCO}^{+}$absorption at $V_{\mathrm{LSR}}=+9 \mathrm{~km} \mathrm{~s}^{-1}$, close to the systemic velocity of Taurus (Hogerheijde et al. 1995; Lucas \& Liszt 1996). No such lines are present toward 3C 273 or 3C 454.3 .

The interferometer data were edited in the usual manner by flagging data points with clearly deviating amplitudes and phases. Editing was especially necessary for daytime observations at $110 \mathrm{GHz}$, when the phase stability of the atmosphere can be low. The data were cleaned using natural weighting and, for some of the ${ }^{13} \mathrm{CO}$ and $\mathrm{C}^{18} \mathrm{O}$ data, a 4" FWHM convolving beam to suppress the noise; this corresponds to a $50 \% u-v$ taper at $45 \mathrm{k} \lambda$. The resulting beam sizes are typically $\sim 5^{\prime \prime}$; the rms noise levels are $0.05-$ $0.1 \mathrm{Jy}$ beam $^{-1}$ per $125 \mathrm{kHz}$ channel. Reduction and analysis of the visibility data were carried out within the MIRIAD software package.

\subsection{Single-Dish Observations}

Single-dish observations of low- and mid- $J$ lines of ${ }^{12} \mathrm{CO}$, ${ }^{13} \mathrm{CO}$, and $\mathrm{C}^{18} \mathrm{O}$ were obtained between 1994 December and 1997 October with the James Clerk Maxwell Telescope $(\mathrm{JCMT}){ }^{2}$ the Caltech Submillimeter Observatory (CSO) ${ }^{3}$ and the IRAM $30 \mathrm{~m}$ telescope (see Table 2). The single-dish observations were reduced and analyzed with the CLASS software package.

Observations of $\mathrm{C}^{18} \mathrm{O} \quad 2-1 \quad(219.56040 \mathrm{GHz})$ were obtained with the JCMT in 1994 December and with the IRAM $30 \mathrm{~m}$ telescope in $1995 \mathrm{May}$, in beams of 23" and 12", respectively, and with velocity resolutions of 0.21 and 0.13 $\mathrm{km} \mathrm{s}^{-1}$. The local oscillator of the JCMT $230 \mathrm{GHz}$ receiver has no phase-lock loop, resulting in a minimum effective line width of $\sim 0.5-1.0 \mathrm{~km} \mathrm{~s}^{-1}$. The observations were made using a position switch between $15^{\prime}$ and $30^{\prime}$ in right ascension, ensuring emission-free offset positions. Pointing accuracy is estimated to be $\sim 5^{\prime \prime}$. Spectra were converted to the main-beam antenna temperature scale using $\eta_{\mathrm{mb}}=0.69$ for the JCMT and $\eta_{\mathrm{mb}}=0.47$ for the IRAM $30 \mathrm{~m}$ spectra. Typical rms noise levels are $0.1-0.3 \mathrm{~K}$.

Using the JCMT, observations of ${ }^{13} \mathrm{CO}(330.58801 \mathrm{GHz})$ 3-2, $\mathrm{C}^{18} \mathrm{O} 3-2$ (329.33057 GHz), and ${ }^{12} \mathrm{CO} 4-3$ (461.04077 $\mathrm{GHz}$ ) were obtained in 1994 December. The FWHM beam size of the JCMT at these frequencies is $14^{\prime \prime}(330 \mathrm{GHz})$ and $11^{\prime \prime}(460 \mathrm{GHz})$. Data were acquired in a position-switched mode with a switch of $15^{\prime}-30^{\prime}$, similar to that for $\mathrm{C}^{18} \mathrm{O} 2-1$. Pointing was checked regularly, and the residual positional uncertainty is less than $5^{\prime \prime}$. The spectra were obtained with the Digital Autocorrelation Spectrometer (DAS) back end, with a typical resolution of $0.1-0.2 \mathrm{~km} \mathrm{~s}^{-1}$. As for the 230 $\mathrm{GHz}$ observations, the absence of a phase-lock loop in the $460 \mathrm{GHz}$ receiver results in an effective line width of $0.5-1.0$

\footnotetext{
${ }^{2}$ The James Clerk Maxwell Telescope is operated by the Joint Astronomy Centre, on behalf of the Particle Physics and Astronomy Research Council of the United Kingdom, the Netherlands Organization for Scientific Research, and the National Research Council of Canada.

${ }^{3}$ The Caltech Submillimeter Observatory is operated by the California Institute of Technology under funding from the U.S. National Science Foundation (AST 93-13929).
} 
TABLE 2

OVERVIEW OF OBSERVATIONS

\begin{tabular}{|c|c|c|c|}
\hline Date & Instrument & Molecular Transition & Sources \\
\hline 1992 Apr, 1993 Jul & OVRO & $\mathrm{HCO}^{+} 1-0$ & $\mathrm{~T} \mathrm{Tau}{ }^{\mathrm{a}}$ \\
\hline 1993 Oct, 1994 Feb-Apr...... & OVRO & $\mathrm{HCO}^{+} 1-0$ & Full sample, except T Tau \\
\hline 1993 Jan, Jun ............... & OVRO & ${ }^{13} \mathrm{CO} / \mathrm{C}^{18} \mathrm{O} 1-0$ & T Tau \\
\hline 1995 Feb-May ................ & OVRO & ${ }^{13} \mathrm{CO} / \mathrm{C}^{18} \mathrm{O} 1-0$ & Full sample, except L1489 IRS, T Tau, TMC 1A \\
\hline 1996 Oct, Nov ................. & OVRO & ${ }^{13} \mathrm{CO} / \mathrm{C}^{18} \mathrm{O} 1-0$ & L1489 IRS, L1551 IRS 5, L1535 IRS, TMC 1A \\
\hline 1997 Feb ....................... & OVRO & ${ }^{13} \mathrm{CO} / \mathrm{C}^{18} \mathrm{O} 1-0$ & L1489 IRS, TMC 1A \\
\hline 1994 Dec ................... & $\mathrm{CSO}$ & ${ }^{12} \mathrm{CO},{ }^{13} \mathrm{CO} 6-5$ & Full sample \\
\hline 1994 Dec ........................ & JCMT & ${ }^{12} \mathrm{CO} 4-3 ;{ }^{13} \mathrm{CO}, \mathrm{C}^{18} \mathrm{O} 3-2$ & Full sample \\
\hline 1995 Мay....................... & IRAM $30 \mathrm{~m}$ & $\mathrm{C}^{18} \mathrm{O} 2-1$ & Full sample \\
\hline 1995 Aug, Oct .................. & JCMT & ${ }^{12} \mathrm{CO} \mathrm{3-2}$ & Full sample, except T Tau, L1551 IRS 5, L1527 IRS \\
\hline 1997 Oct ...................... & JCMT & ${ }^{13} \mathrm{CO} \mathrm{3-2}$ & L1489 IRS, TMR 1, TMC 1A, L1527 IRS \\
\hline
\end{tabular}

${ }^{\text {a }}$ Previously presented by van Langevelde et al. 1994a.

$\mathrm{km} \mathrm{s}^{-1}$ at this frequency. The spectra were converted to the main-beam antenna temperature scale using $\eta_{\mathrm{mb}}=0.58$ $(330 \mathrm{GHz})$ and $0.48(460 \mathrm{GHz})$, obtained from measurements of Jupiter and Mars by the JCMT staff. Typical rms noise levels were $0.1 \mathrm{~K}$ at $330 \mathrm{GHz}$ and $0.4 \mathrm{~K}$ at $460 \mathrm{GHz}$. Higher signal-to-noise ratio observations of ${ }^{13} \mathrm{CO} 3-2$ were obtained on 1997 October 30 toward four sources, using the JCMT and a similar observational setup.

Maps covering $2^{\prime} \times 2^{\prime}$ in ${ }^{12} \mathrm{CO} 3-2(345.79599 \mathrm{GHz})$ of L1489 IRS, Haro 6-10, L1535 IRS, TMR 1, TMC 1A, and TMC 1 were made with the JCMT in the "on-the-fly" mapping mode in 1995 August and October. In this mode, the telescope is scanned constantly in one direction, taking short integrations of typically $5 \mathrm{~s}$ every $3^{\prime \prime}$. Only one integration at an off position (typically $30^{\prime}$ away) is used to calibrate a complete row. The adopted off positions were checked for the presence of emission; in a few cases emission appeared to be present, and the maps were corrected with a high signal-to-noise ratio spectrum obtained with a larger position switch. The total map is built up of the required number of rows, separated by half a beam (6"), resulting in fully sampled maps. Multiple maps are stacked to arrive at the final noise level. Pointing was checked regularly during the observations; the positional accuracy of the maps was estimated to be better than $5^{\prime \prime}$. The final data have a typical main-beam rms noise level of $\sim 0.25 \mathrm{~K}$ per point in $1 \mathrm{~km}$ $\mathrm{s}^{-1}$ wide channels, using $\eta_{\mathrm{mb}}=0.58$.

It is found (see $\S 4$ ) that the observed outflows are less than $1^{\prime}$ in extent. To ensure that no outflow emission was missed by the $2^{\prime} \times 2^{\prime}$ maps, $6^{\prime}$ long strips at the approximate position angle of the outflows were observed on-the-fly in ${ }^{12} \mathrm{CO} 3-2$. For comparison, a similar strip has been observed toward L1527 IRS, for which outflow emission in ${ }^{12} \mathrm{CO} 3-2$ over 6 was observed by MacLeod et al. (1994). For completeness, the ${ }^{12} \mathrm{CO} 3-2$ data presented by these authors have been taken from the JCMT data archive and are analyzed here alongside our own data. A $1^{\prime} \times 1^{\prime}$ map of $\mathrm{T}$ Tau in ${ }^{12} \mathrm{CO} 3-2$, obtained with the CSO in 1989 December with a beam efficiency of 0.56 and a beam size of $30^{\prime \prime}$, is also reproduced here for completeness.

In 1994 December, observations of ${ }^{12} \mathrm{CO}(691.471978$ $\mathrm{GHz})$ and ${ }^{13} \mathrm{CO}$ 6-5 (661.067375 GHz) were obtained toward all sources with the CSO under excellent weather conditions (typical system temperatures of $1500 \mathrm{~K}$ at 690 $\mathrm{GHz}$ ). The spectra were obtained with a position switch of $10^{\prime}$ and recorded with the facility $50 \mathrm{MHz}$ and $500 \mathrm{MHz}$ bandwidth Acousto-Optical Spectrometers (AOSs). Pointing was checked regularly, and found to vary by up to $5^{\prime \prime}-10^{\prime \prime}$. An additional source of positional error was the correction for the atmospheric diffraction, which is comparable to the FWHM beam size at these frequencies $\left(\sim 10^{\prime \prime}\right)$. It is estimated that the pointing is no better than $\sim 10^{\prime \prime}$. The spectra were converted to the main-beam antenna scale using $\eta_{\mathrm{mb}}=0.35$, obtained from measurements of Mars, Saturn, and the Moon (J. Keene 1995, private communication). The resulting rms noise level was $0.4 \mathrm{~K}$ in $0.2 \mathrm{~km} \mathrm{~s}^{-1}$ wide channels (AOS $50 \mathrm{MHz}$, binned), after a typical integration time of only 5 minutes. Toward T Tau, a combined setting of ${ }^{13} \mathrm{CO}$ and $\mathrm{C}^{18} \mathrm{O} 6-5(658.553325 \mathrm{GHz})$ was also observed. However, technical difficulties prevented measurement of the sideband ratio, leaving the calibration highly uncertain because of the proximity of a telluric $\mathrm{O}_{3}$ $Q$-branch.

\section{INTERFEROMETER OBSERVATIONS: MOLECULAR MASS AND $\mathrm{HCO}^{+}$ABUNDANCE}

Compact emission in $\mathrm{HCO}^{+},{ }^{13} \mathrm{CO}$, and $\mathrm{C}^{18} \mathrm{O} 1-0$ is detected in the OVRO beam toward all sources, as is shown in the integrated intensity images of Figure 1 . The emission is resolved, with the highest fluxes recovered on baselines $<10 \mathrm{k} \lambda$. Image reconstruction in $\mathrm{HCO}^{+}$and ${ }^{13} \mathrm{CO}$ is complicated by the missing zero-spacing flux, especially toward $\mathrm{T}$ Tau and L1551 IRS 5. Figure 2 shows the spectra obtained in the $\sim 5^{\prime \prime}$ FWHM synthesized beams toward the source positions. For T Tau, the $\mathrm{HCO}^{+} 3-2$ spectrum from Hogerheijde et al. (1997b) is also presented. The details of the spatial and kinematic structure of the aperture synthesis results of the individual sources can most easily be appreciated in conjunction with the observations of the outflows, the discussion of which is postponed until $\S 5.1$. Here, we will derive general characteristics of the emission, such as the molecular mass, the $\mathrm{HCO}^{+}$abundance on the probed scales, and the fraction of recovered flux.

The velocity-integrated line fluxes are listed in Table 3, averaged over areas of $5^{\prime \prime} \times 5^{\prime \prime}$ and $20^{\prime \prime} \times 20^{\prime \prime}$, corresponding to the synthesized beam and to the typical single-dish beam of the $\mathrm{HCO}^{+}$observations of Paper I and the $\mathrm{CO}$ observations presented here, respectively. Only pixels with a signal of $\geq 3 \sigma$ are included in the average, and the fluxes have been transformed to brightness temperatures $T_{b}$. $\mathrm{C}^{18} \mathrm{O}$ emission is undetected at the obtained noise level toward Haro 6-10 and L1527 IRS, and toward TMC 1A over the $20^{\prime \prime} \times 20^{\prime \prime}$ area. Over the $5^{\prime \prime} \times 5^{\prime \prime}$ area, $\mathrm{HCO}^{+}$is undetected toward Haro 6-10 and toward L1527 IRS, where this region coincides with the resolved-out center of the cross-shaped emission seen in Figure 1 (see also $\S$ 5.1.8). Assuming an 

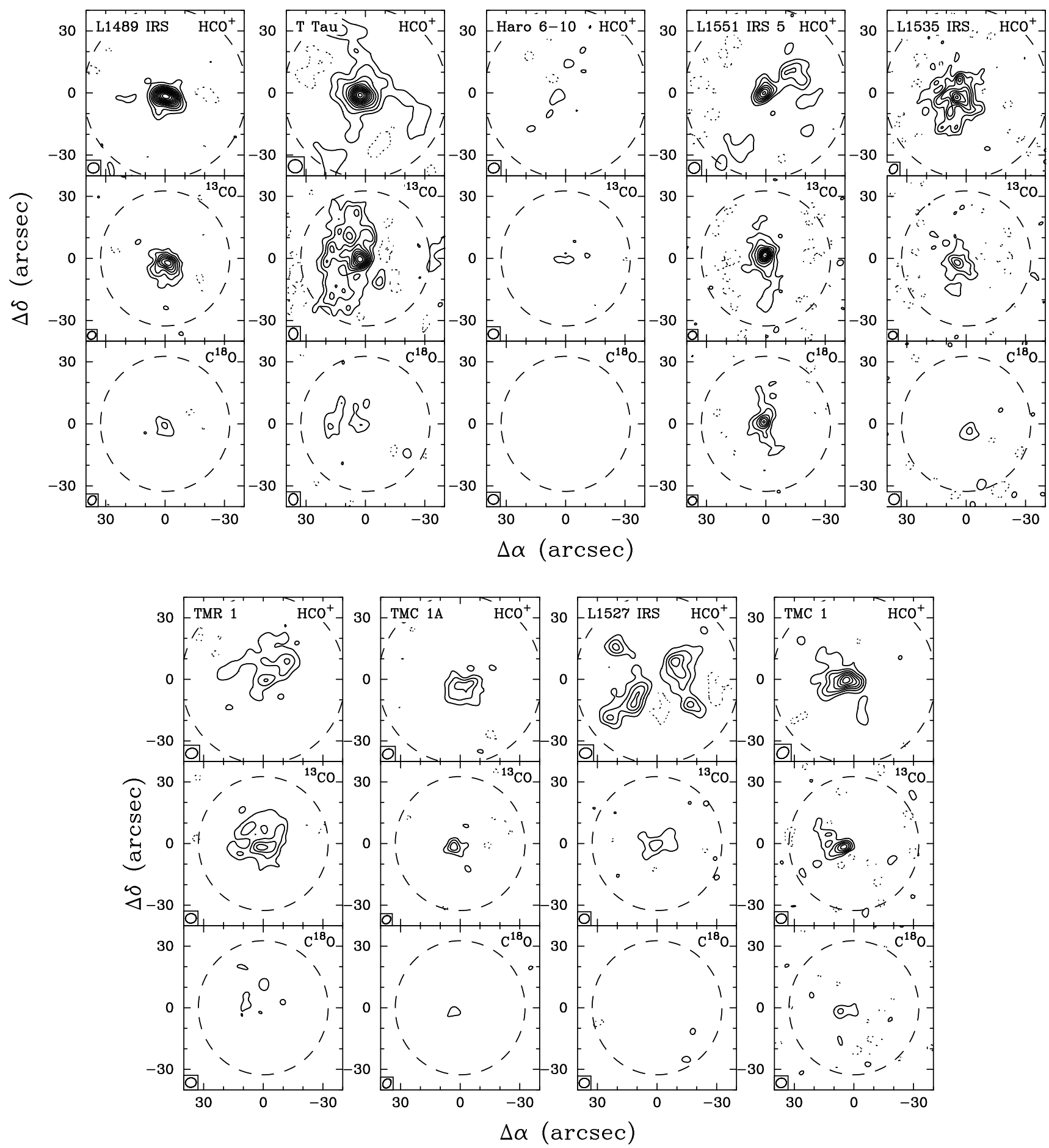

FIG. 1.- Integrated $\mathrm{HCO}^{+}$(top),${ }^{13} \mathrm{CO}$ (middle), and $\mathrm{C}^{18} \mathrm{O}$ (bottom) 1-0 emission observed with the OVRO millimeter array. Contours are drawn at intervals of $3 \sigma \approx 0.06-0.12 \mathrm{Jy}$ beam $^{-1}$. The naturally weighted, synthesized beams of FWHM $\sim 5^{\prime \prime}$ are shown in the lower left corner of each panel. The OVRO primary beam is indicated by the dashed circle.

abundance ratio of 8 for $\left[{ }^{13} \mathrm{CO}\right]:\left[\mathrm{C}^{18} \mathrm{O}\right]$, the ${ }^{13} \mathrm{CO}$ lines are found to be at most moderately optically thick, with velocity-averaged opacities of $\bar{\tau} \lesssim 6$; the $\mathrm{C}^{18} \mathrm{O}$ lines are always optically thin. Estimates of the opacity of the $\mathrm{HCO}^{+}$ lines are taken from the single-dish $\mathrm{HCO}^{+}$and $\mathrm{H}^{13} \mathrm{CO}^{+}$ 1-0 data of Paper I and Mizuno et al. (1994). In contrast to Paper I, a beam efficiency of 0.8 is used here for the $\mathrm{H}^{13} \mathrm{CO}^{+}$Nobeyama data (cf. Kitamura et al. 1990), resulting in opacities increased by a factor of 1.25 compared to Table 6 of Paper I. No $\mathrm{H}^{13} \mathrm{CO}^{+}$data are available for four sources, and the median value of $\tau=15$ is used for these. This opacity correction is only approximate, since the single-dish values can differ significantly from the opacity of the material traced in the interferometer. Aperture-synthesis $\mathrm{H}^{13} \mathrm{CO}^{+}$observations are required to place firmer limits on the opacity.

Molecular masses can be estimated from the optically thin $\mathrm{C}^{18} \mathrm{O}$ images, or from the ${ }^{13} \mathrm{CO}$ data, taking into account the allowed $\bar{\tau}$ range. Assuming an excitation temperature $T_{\text {ex }}$, the average column density is given in cgs 

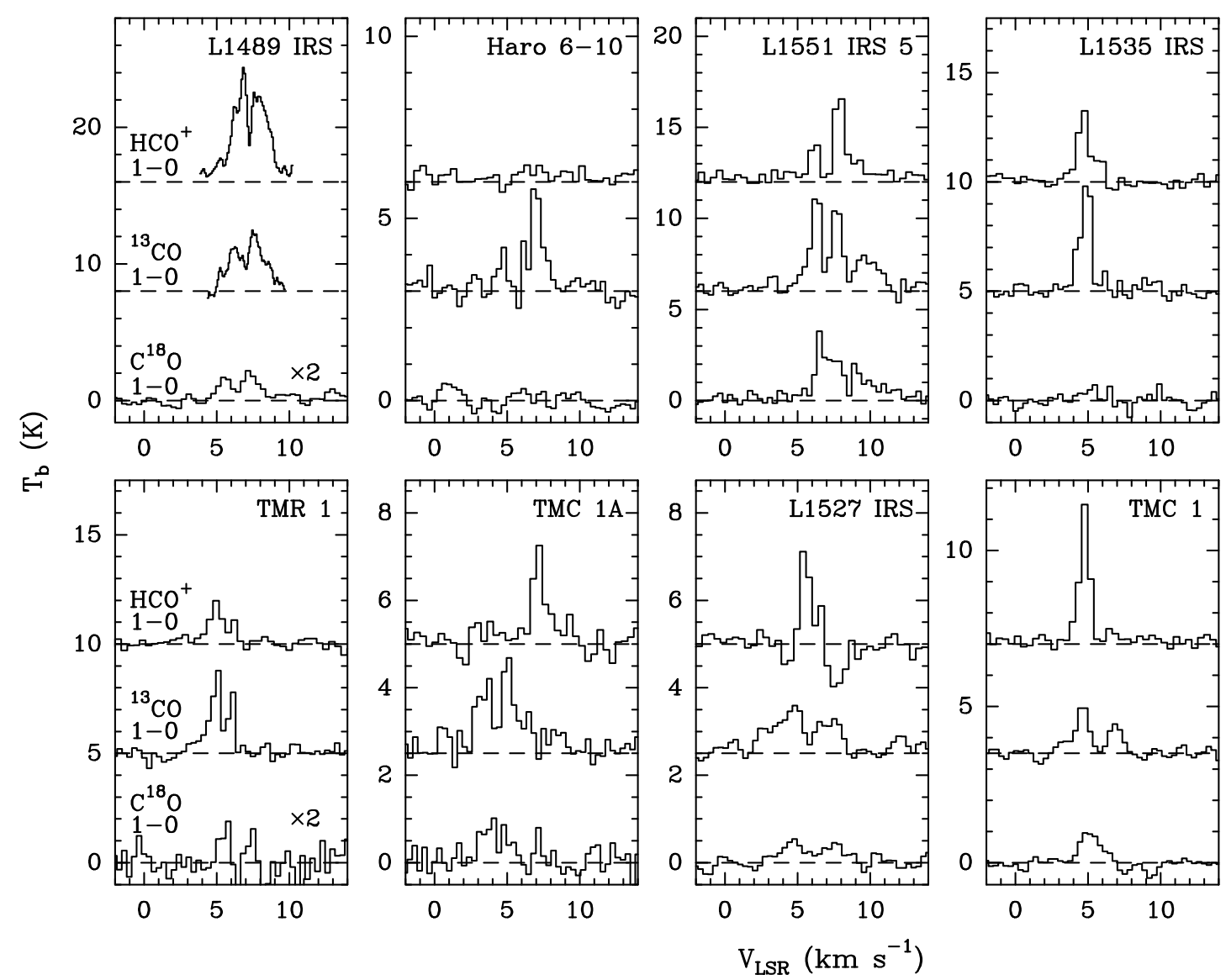

Spectra in synthesized OVRO beam

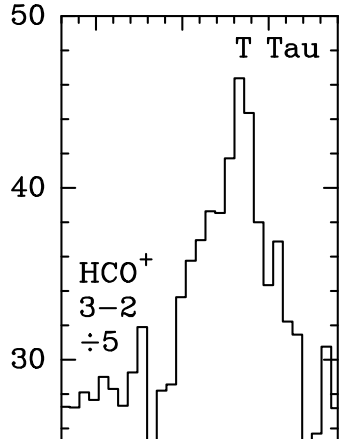

FIG. 2.-Spectra of $\mathrm{HCO}^{+},{ }^{13} \mathrm{CO}$, and $\mathrm{C}^{18} \mathrm{O} 1-0$ emission observed with OVRO in a $5^{\prime \prime} \times 5^{\prime \prime}$ region around the continuum position. For T Tau, the $\mathrm{HCO}^{+}$3-2 spectrum observed by Hogerheijde et al. (1997a) is also shown. The intensity scale has been converted to brightness temperature; some spectra are scaled by the indicated factors for clarity.

units by

$$
\begin{aligned}
\bar{N}=10^{5} \times \frac{3 k^{2}}{4 h \pi^{3} \mu^{2} v^{2}} \exp \left(\frac{h v J_{l}}{2 k T_{\mathrm{ex}}}\right) \\
\times \frac{T_{\mathrm{ex}}+h v / 6 k\left(J_{l}+1\right)}{e^{-h v / k T_{\mathrm{ex}}}} \int T_{b}\left(\frac{\tau}{1-e^{-\tau}}\right) d V
\end{aligned}
$$

(Scoville et al. 1986), where $\int T_{b} d V$ is the integrated brightness temperature in $\mathrm{K} \mathrm{km} \mathrm{s}^{-1}$ of the $J_{u}-J_{l}$ transition with frequency $v$, and $\mu$ is the permanent dipole $(0.112 \mathrm{D}$ for $\mathrm{C}^{18} \mathrm{O}$ and ${ }^{13} \mathrm{CO}$, and $3.91 \mathrm{D}$ for $\mathrm{HCO}^{+}$; Millar et al. 1991). The single-dish $\mathrm{HCO}^{+}$and $\mathrm{H}^{13} \mathrm{CO}^{+}$data of Paper I and the $\mathrm{C}^{18} \mathrm{O}$ data presented here indicate excitation temperatures of $20-80 \mathrm{~K}$ for $\mathrm{HCO}^{+}$and $\mathrm{C}^{18} \mathrm{O}$, as compared to escape probability calculations. A number of observational uncertainties exist in the derivation of the excitation temperature of individual sources, and the material traced in the OVRO beam may have a different excitation. Adopting $T_{\text {ex }}=40 \mathrm{~K}$ as a fiducial average value, equation (1) reduces to $\bar{N}=2.13 \times 10^{15} \int T_{b}[\tau / 1-\exp (-\tau)] d V$ in $\mathrm{cm}^{-2}$ for ${ }^{13} \mathrm{CO}$ and $\mathrm{C}^{18} \mathrm{O}$, and to $\bar{N}=2.59 \times 10^{12} \int T_{b}[\tau / 1-\exp$ $(-\tau)] d V$ for $\mathrm{HCO}^{+}$. Using a mean molecular weight of $2.4 m_{\mathrm{H}}$, and the standard abundance ratios of $\left[{ }^{12} \mathrm{CO}\right]:\left[{ }^{13} \mathrm{CO}\right]=65: 1$ (cf. Langer \& Penzias 1990), $\left[{ }^{12} \mathrm{CO}\right]:\left[{ }^{18} \mathrm{CO}\right]=500: 1$, and $\left[{ }^{12} \mathrm{CO}\right]:\left[\mathrm{H}_{2}\right]=1: 10^{4}$, the molecular mass is given by $M / M_{\odot}=2.3 \times 10^{-3}$ $\int T_{b}[\tau / 1-\exp (-\tau)] d V\left(5^{\prime \prime} \times 5^{\prime \prime}\right)$ or $3.8 \times 10^{-2} \int T_{b}[\tau / 1-$ $\exp (-\tau)] d V\left(20^{\prime \prime} \times 20^{\prime \prime}\right)$ for $\mathrm{C}^{18} \mathrm{O}$. For ${ }^{13} \mathrm{CO}$, these factors are divided by the abundance ratio of 8 . The derived masses are listed in Table 3, with an uncertainty of a factor of $\sim 2$ due to the spread in $T_{\text {ex }}$. They agree well with other estimates (see below and $\S 5$ ).

From the opacity-corrected column densities, the $\mathrm{HCO}^{+}$ abundance is inferred and listed in Table 8 . The uncertainty in $T_{\text {ex }}$ largely drops out of the abundance, provided that $\mathrm{C}^{18} \mathrm{O}$ and $\mathrm{HCO}^{+}$have similar excitation temperatures. An average abundance with respect to $\mathrm{H}_{2}$ of $4 \times 10^{-8}$ is found over the $5^{\prime \prime} \times 5^{\prime \prime}$ and $20^{\prime \prime} \times 20^{\prime \prime}$ regions, with a spread of a factor of 3 between the different sources. Compared to the $15^{\prime \prime}-19^{\prime \prime}$ single-dish value of $(5.0 \pm 1.7) \times 10^{-9}$ from Paper I, including the different beam efficiencies used here (see $\S 1$ ), this value is larger on average by a factor of 8 , and by $0.64-46$ for individual sources. This may indicate that the $\mathrm{HCO}^{+}$abundance is increased by factors of a few on the small scales sampled by the interferometer. Part of this increase can be explained by the assumption of equal excitation temperatures for $\mathrm{C}^{18} \mathrm{O}$ and $\mathrm{HCO}^{+}$, as well as by contributions from extended $\mathrm{C}^{18} \mathrm{O}$ emission to the single-dish lines. The interferometer data as well as the $\mathrm{H}^{13} \mathrm{CO}^{+}$singledish data are likely to exclusively trace the YSO envelopes. Because of the approximate nature of the opacity correction of the $\mathrm{HCO}^{+}$fluxes, interferometer observations of $\mathrm{H}^{13} \mathrm{CO}^{+}$are required to firmly constrain the abundance.

Single-dish and interferometer observations can be fully integrated by adding zero-spacing fluxes to the visibility data (see, e.g., Wilner \& Welch 1994; Velusamy, Kuiper, \& Langer 1995; Zhou, Evans, \& Wang 1996 for recent applications of this technique to YSO observations). However, 
TABLE 3

OVRO INTEGRated Intensities, Molecular Masses, and $\mathrm{HCO}^{+}$Abundances

\begin{tabular}{|c|c|c|c|c|c|c|c|}
\hline \multirow[b]{2}{*}{ SOURCE } & \multicolumn{3}{|c|}{$\int T_{b} d V$} & \multirow[b]{2}{*}{$\bar{\tau}\left({ }^{13} \mathrm{CO}\right)$} & \multirow[b]{2}{*}{$\bar{\tau}\left(\mathrm{HCO}^{+}\right)^{\mathrm{a}}$} & \multirow[b]{2}{*}{$\begin{array}{c}M_{\text {mol }}^{\mathrm{b}} \\
\left(10^{-3} M_{\odot}\right)\end{array}$} & \multirow[b]{2}{*}{$X\left(\mathrm{HCO}^{+}\right)$} \\
\hline & $\left(\begin{array}{c}\left.{ }^{13} \mathrm{CO}^{-1}\right) \\
\left(\mathrm{km} \mathrm{s}^{-1}\right)\end{array}\right.$ & $\begin{array}{c}\mathrm{C}^{18} \mathrm{O} \\
\left(\mathrm{K} \mathrm{km} \mathrm{s}^{-1}\right)\end{array}$ & $\begin{array}{c}\mathrm{HCO}^{+} \\
\left(\mathrm{K} \mathrm{km} \mathrm{s}^{-1}\right)\end{array}$ & & & & \\
\hline \multicolumn{8}{|c|}{ Average over $5^{\prime \prime} \times 5^{\prime \prime}$ area } \\
\hline L1489 IRS . & $12.9 \pm 0.8$ & $2.7 \pm 0.5$ & $20.7 \pm 0.7$ & $1.4 \pm 0.8$ & 15 & $5.8 \pm 1.1$ & $(2.8 \pm 0.6) \times 10^{-8}$ \\
\hline Т Tau ...... & $11.9 \pm 0.6$ & $1.4 \pm 0.3$ & $19.0 \pm 0.4$ & $<\overline{0} .4$ & 15 & $3.0 \pm 0.7$ & $(5.0 \pm 1.2) \times 10^{-8}$ \\
\hline Haro 6-10 & $2.4 \pm 0.3$ & $<0.8$ & $<\overline{1.2}$ & $<3.7$ & 15 & $0.6-2.7$ & \\
\hline L1551 IRS $5 \ldots \ldots$ & $18.6 \pm 0.9$ & $9.8 \pm 0.8$ & $8.5 \pm 0.5$ & $6.1 \pm 1.2$ & 15.6 & $31.0 \pm 1.7$ & $(3.3 \pm 0.3) \times 10^{-9}$ \\
\hline L1535 IRS . & $5.9 \pm 0.6$ & $0.4 \pm 0.1$ & $13.1 \pm 0.7$ & $<0.2$ & 11.2 & $0.9 \pm 0.2$ & $(8.9 \pm 2.3) \times 10^{-8}$ \\
\hline TMR $1 \ldots$ & $5.0 \pm 0.5$ & $0.5 \pm 0.3$ & $3.6 \pm 0.6$ & $<0.9$ & 15.4 & $1.0 \pm 0.7$ & $(2.9 \pm 2.1) \times 10^{-8}$ \\
\hline TMC 1A.. & $6.2 \pm 0.8$ & $1.6 \pm 0.7$ & $3.5 \pm 0.4$ & $2.1 \pm 0.2$ & 27.4 & $1.7 \pm 0.2$ & $(1.4 \pm 0.7) \times 10^{-8}$ \\
\hline L1527 IRS ........ & $4.1 \pm 0.6$ & $<1.9$ & $<\overline{2.0}$ & $<6.2$ & 23.4 & $0.9-6.8$ & \\
\hline TMC $1 \ldots \ldots \ldots \ldots$ & $1.6 \pm 0.2$ & $0.7 \pm 0.2$ & $4.2 \pm 0.2$ & $5.3 \pm 3.0$ & 15 & $2.2 \pm 0.5$ & $(2.1 \pm 0.7) \times 10^{-8}$ \\
\hline \multicolumn{8}{|c|}{ Average over $20^{\prime \prime} \times 20^{\prime \prime}$ area } \\
\hline L1489 IRS . & $3.6 \pm 0.2$ & $0.34 \pm 0.13$ & $5.5 \pm 0.2$ & $<0.2$ & 15 & $13.0 \pm 0.9$ & $(5.9 \pm 2.3) \times 10^{-8}$ \\
\hline T Tau .... & $4.1 \pm 0.1$ & $0.23 \pm 0.08$ & $7.5 \pm 0.1$ & $<0.2$ & 15 & $8.6 \pm 3.0$ & $(1.2 \pm 0.4) \times 10^{-7}$ \\
\hline Haro 6-10 & $0.2 \pm 0.1$ & $<\overline{0.21}$ & $0.2 \pm 0.1$ & $\ldots$ & 15 & $0.5-3.0$ & $(0.9-5.3) \times 10^{-8}$ \\
\hline L1551 IRS $5 \ldots \ldots$ & $4.7 \pm 0.2$ & $2.22 \pm 0.18$ & $1.8 \pm 0.1$ & $5.2 \pm 0.9$ & 15.6 & $113.0 \pm 6.8$ & $(3.0 \pm 0.3) \times 10^{-9}$ \\
\hline L1535 IRS ..... & $1.6 \pm 0.2$ & $0.06 \pm 0.03$ & $6.3 \pm 0.2$ & $<\overline{0} .2$ & 11.2 & $2.3 \pm 1.1$ & $(2.8 \pm 1.4) \times 10^{-7}$ \\
\hline TMR $1 \ldots$. & $2.3 \pm 0.1$ & $0.24 \pm 0.08$ & $1.5 \pm 0.1$ & $<0.4$ & 15.4 & $9.0 \pm 3.0$ & $(2.3 \pm 0.8) \times 10^{-8}$ \\
\hline TMC $1 \mathrm{~A} \ldots .$. & $0.8 \pm 0.2$ & $<0.56$ & $1.6 \pm 0.1$ & $(<22)$ & 27.4 & $3.9 \pm 0.9$ & $(0.5-1.3) \times 10^{-7}$ \\
\hline L1527 IRS .. & $1.1 \pm 0.2$ & $<0.46$ & $2.3 \pm 0.2$ & $<5.7$ & 23.4 & $4.6-30.2$ & $(1.6-9.2) \times 10^{-8}$ \\
\hline TMC $1 \ldots \ldots \ldots \ldots$ & $0.4 \pm 0.1$ & $14 \pm 0.06$ & $1.4 \pm 0.1$ & $4.7 \pm 4.1$ & 15 & $7.2 \pm 2.3$ & $(3.6 \pm 1.6) \times 10^{-8}$ \\
\hline
\end{tabular}

${ }^{\text {a }}$ From Paper I, but correcting for a beam efficiency of 0.8 for the $\mathrm{H}^{13} \mathrm{CO}^{+}$Nobeyama data (Mizuno et al. 1994). For L1489 IRS, T Tau, Haro 6-10, and TMC 1, the median value of $\tau=15$ is assumed.

${ }^{\mathrm{b}}$ Molecular mass derived from $\mathrm{C}^{18} \mathrm{O}$ or ${ }^{13} \mathrm{CO}$ using equation (1) with $T_{\mathrm{ex}}=40 \mathrm{~K}$ and correcting for line opacity. Listed uncertainties in the mass only include the observational noise; the total uncertainty amounts to a factor of $\sim 2$ from the range in inferred excitation temperatures (see $\S 3$ ). Upper limits on $\mathrm{C}^{18} \mathrm{O}$ result in a range in inferred mass and $X\left(\mathrm{HCO}^{+}\right)$.

many important aspects can be obtained by a simple, direct comparison of the data sets. In Figure 3, the $\mathrm{HCO}^{+} 1-0$ OVRO images and the $\mathrm{HCO}^{+} 3-2$ map of T Tau from Hogerheijde et al. (1997b) are superposed on the single-dish $\mathrm{HCO}^{+}$1-0, 3-2, and 4-3 data of Paper I. The compact $\mathrm{HCO}^{+} 1-0$ is seen to trace the peak of single-dish emission, with a size comparable to the 3-2 and 4-3 cores at $14^{\prime \prime}-19^{\prime \prime}$ resolution. This supports the conclusion from Paper I that the latter provide a reliable probe of the inner envelopes. The single-dish $\mathrm{HCO}^{+} 1-0$ emission around L1551 IRS 5 lacks strong central concentration, and only a number of $\mathrm{HCO}^{+}$clumps are recovered in the OVRO beam. Toward Haro 6-10, almost all $\mathrm{HCO}^{+}$is resolved out by the interferometer. In Figure 4, the $\mathrm{HCO}^{+}$line profiles from the $28^{\prime \prime}$ IRAM $30 \mathrm{~m}$ beam are compared to the OVRO data, after convolving to the same resolution. Toward some sources (L1489 IRS, TMR 1, L1527 IRS, TMC 1) comparable line profiles are seen, while toward, e.g., L1551 IRS 5, only redshifted emission is recovered. A deep absorption dip observed by OVRO in $\mathrm{HCO}^{+} 1-0$ toward T Tau is filled in by large-scale emission in the IRAM beam (van Langevelde et al. 1994a).

In Table 4, the $\mathrm{HCO}^{+}$and $\mathrm{C}^{18} \mathrm{O}$ fluxes integrated over $28^{\prime \prime}$ and $20^{\prime \prime}$ regions, respectively, are listed as fractions of the flux obtained in single-dish beams of similar size $\left(\mathrm{HCO}^{+}\right.$from Paper I; $\mathrm{C}^{18} \mathrm{O}$ from Hayashi et al. 1994, adopting $\eta_{\mathrm{mb}}=0.4$ ). In $\mathrm{HCO}^{+}$, the OVRO beam traces $25 \%-50 \%$ of the single-dish flux, except in Haro 6-10 (3\%) and L1551 IRS 5 (11\%). Lower fractions of $\lesssim 20 \%$ are recovered in $\mathrm{C}^{18} \mathrm{O}$. This indicates that $\mathrm{HCO}^{+}$emission is predominantly associated with compact structure in the envelopes, possibly because of an increased abundance on these scales, while the surrounding cloud contributes signifi- cantly to the single-dish $\mathrm{C}^{18} \mathrm{O}$ flux. The cloud core around Haro 6-10 appears to lack any central, compact condensation, since only marginal emission is detected in the OVRO beam from $\mathrm{HCO}^{+},{ }^{13} \mathrm{CO}$, or $\mathrm{C}^{18} \mathrm{O}$, while the low $\mathrm{HCO}^{+}$flux recovered toward L1551 IRS 5 is likely to be due to the opacity of the surrounding cloud, because as much as $42 \%$ of the single-dish flux is recovered in the optically thin $\mathrm{C}^{18} \mathrm{O}$ line. This fraction is large compared to the other sources, suggesting that L1551 IRS 5 has a relatively massive inner envelope. From $\mathrm{H}^{13} \mathrm{CO}^{+}$interferometer observations, Saito et al. (1996) find a mass of $0.27 M_{\odot}$. In the last column of Table 4, the derived molecular masses are compared to the values obtained from single-dish 1.1

TABLE 4

Flux and Mass Ratios OVRO versus Single-Dish

\begin{tabular}{|c|c|c|c|}
\hline Source & $\begin{array}{c}\mathrm{HCO}^{+\mathrm{a}, \mathrm{d}} \\
(\%)\end{array}$ & $\begin{array}{c}\mathrm{C}^{18} \mathrm{O}^{\mathrm{b}, \mathrm{d}} \\
(\%)\end{array}$ & $\begin{array}{c}\text { Mass }^{\mathrm{c}, \mathrm{d}} \\
(\%)\end{array}$ \\
\hline L1489 IRS & $38 \pm 15$ & $15 \pm 6$ & 52-81 \\
\hline T Tau . & $26 \pm 10$ & $13 \pm 5$ & $30 \pm 12$ \\
\hline Haro 6-10 & $3 \pm 1$ & $<4$ & $>12$ \\
\hline L1551 IRS $5 \ldots \ldots$ & $11 \pm 4$ & $42 \pm 17$ & $43 \pm 17$ \\
\hline L1535 IRS ......... & $51 \pm 20$ & $<21$ & $>64$ \\
\hline TMR $1 \ldots \ldots \ldots \ldots$ & $29 \pm 12$ & $6 \pm 3$ & $129 \pm 50$ \\
\hline TMC 1A. & $35 \pm 14$ & $<22$ & $22 \pm 9$ \\
\hline L1527 IRS . & $41 \pm 16$ & $<14$ & $15-97$ \\
\hline TMC $1 \ldots .$. & $37 \pm 15$ & $3 \pm 2$ & 45-144 \\
\hline
\end{tabular}

${ }^{a}$ Ratio of OVRO (28" area) over IRAM $30 \mathrm{~m}$ (28" beam; Paper I).

${ }^{b}$ Ratio of OVRO (20" area) over Nobeyama (16" beam; Hayashi et al. 1994, using $\eta_{\mathrm{mb}}=0.4$ ).

${ }^{\circ}$ Ratio of OVRO (20" area) over mass from $\lambda=1.1 \mathrm{~mm}$ dust continuum (19" beam; Paper I).

${ }^{d}$ Error based on $20 \%$ accuracy in flux calibration. 


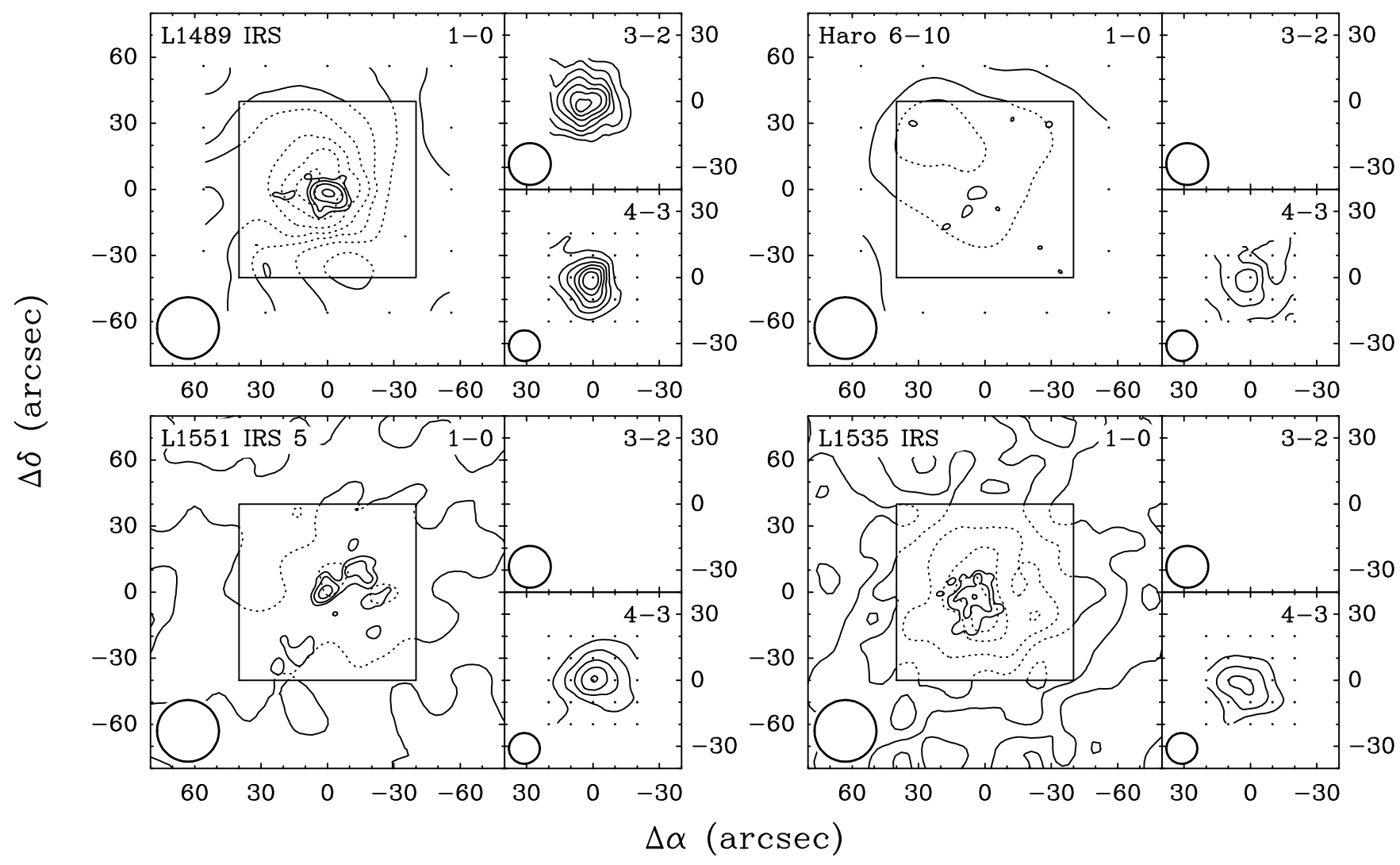

Fig. 3.-Single-dish $\mathrm{HCO}^{+} 1-0,3-2$, and 4-3 maps from Paper I, overlaid with the $\mathrm{HCO}^{+} 1-0$ OVRO images. For T Tau, the $\mathrm{HCO}^{+} 3-2 \mathrm{OVRO}^{-1 m a g e s}$ from Hogerheijde et al. (1997a) are reproduced in the inset. The details of the individual sources are discussed in $\S 5.1$.

mm continuum measurements (Paper I). Toward most sources, the $\mathrm{C}^{18} \mathrm{O}$ OVRO data and the $1.1 \mathrm{~mm}$ continuum fluxes appear to trace the same material; only toward T Tau and TMC $1 \mathrm{~A}$ is the derived mass less than $50 \%$ of the dust value, suggesting an enhanced dust temperature and, consequently, a lower dust mass. The fraction of recovered $\mathrm{HCO}^{+}$and $\mathrm{C}^{18} \mathrm{O}$ flux toward these sources is comparable to what is found for the other sources, supporting the conclusion that the dust mass may have been overestimated by a factor of $\sim 2$ for these two sources.

\section{SINGLE-DISH OBSERVATIONS: PROPERTIES OF THE ENVELOPES AND OUTFLOWS}

\subsection{Line Profiles, Opacities, and Excitation Conditions}

Emission in the observed transitions is detected toward all sources of the sample, with intensities ranging between 1 to a few $\mathrm{K}$ for the $\mathrm{C}^{18} \mathrm{O}$ lines to $20-60 \mathrm{~K}$ for the ${ }^{12} \mathrm{CO} 4-3$ and 6-5 lines. Most notable is the detection of ${ }^{12} \mathrm{CO}$ and ${ }^{13} \mathrm{CO} 6-5$ toward all sources, indicating the presence of appreciable amounts of warm $\left(T_{\text {kin }}>80 \mathrm{~K}\right)$ gas around embedded low-mass YSOs. Previously, emission in this line from YSOs had only been detected toward a small number of T Tauri stars (Schuster et al. 1993; see also Spaans et al. 1995). The ${ }^{12} \mathrm{CO},{ }^{13} \mathrm{CO}$, and $\mathrm{C}^{18} \mathrm{O}$ spectra observed toward the sources are presented in Figure 5; the integrated line intensities are listed in Table 5.

The $\mathrm{C}^{18} \mathrm{O} 2-1$ and 3-2 and ${ }^{13} \mathrm{CO} 6-5$ lines are narrow, and best described by single Gaussians of FWHM 0.75-3.0 $\mathrm{km} \mathrm{s}^{-1}$. The systemic velocity $V_{0}$ of the sources can be accurately determined from these lines (Table 5), and agree to within $0.5 \mathrm{~km} \mathrm{~s}^{-1}$ for the different transitions, often to within the velocity resolution. The ${ }^{12} \mathrm{CO} 3-2,4-3$, and 6-5 line profiles are characterized by extended line wings and

TABLE 5

SINGLE-Dish CO INTEGRATED INTENSITIES $\int T_{\mathrm{mb}} d V$

\begin{tabular}{|c|c|c|c|c|c|c|c|c|}
\hline Source & $\begin{array}{c}V_{0} \\
\left(\mathrm{~km} \mathrm{~s}^{-1}\right)\end{array}$ & $\begin{array}{c}\mathrm{C}^{18} \mathrm{O} 2-1 \\
\left(\mathrm{~K} \mathrm{~km} \mathrm{~s}^{-1}\right)\end{array}$ & $\begin{array}{c}\mathrm{C}^{18} \mathrm{O} \mathrm{3}_{3-2} \\
\left(\mathrm{~K} \mathrm{~km} \mathrm{~s}^{-1}\right)\end{array}$ & $\begin{array}{c}{ }^{13} \mathrm{CO} \mathrm{3-2}^{-2} \\
\left(\mathrm{~K} \mathrm{~km} \mathrm{~s}^{-1}\right)\end{array}$ & $\begin{array}{c}{ }^{12} \mathrm{CO} \mathrm{3}^{-2} \\
\left(\mathrm{~K} \mathrm{~km} \mathrm{~s}^{-1}\right)\end{array}$ & $\begin{array}{c}{ }^{12} \mathrm{CO}_{4-3} \\
\left(\mathrm{~K} \mathrm{~km} \mathrm{~s}^{-1}\right)\end{array}$ & $\begin{array}{c}{ }^{13} \mathrm{CO} \mathrm{6-5}^{6-5} \\
\left(\mathrm{~K} \mathrm{~km} \mathrm{~s}^{-1}\right)\end{array}$ & $\begin{array}{c}{ }^{12} \mathrm{CO}_{6-5} \\
\left(\mathrm{~K} \mathrm{~km} \mathrm{~s}^{-1}\right)\end{array}$ \\
\hline L1489 IRS . & 7.1 & $2.97 \pm 0.12$ & $3.95 \pm 0.18$ & $14.3 \pm 0.37$ & $25.1 \pm 1.2$ & $44.7 \pm 0.40$ & $8.20 \pm 0.52$ & $37.3 \pm 0.73$ \\
\hline Т Тau ...... & 8.0 & $3.63 \pm 0.22$ & $7.08 \pm 0.28$ & $45.8 \pm 0.47$ & & $279.8 \pm 0.80$ & $53.1 \pm 0.40$ & $243.8 \pm 0.73$ \\
\hline Haro 6-10 & 7.0 & $4.25 \pm 0.18$ & $3.58 \pm 0.19$ & $14.8 \pm 0.26$ & $66.2 \pm 2.9$ & $97.5 \pm 0.51$ & $13.4 \pm 0.46$ & $119.5 \pm 0.89$ \\
\hline L1551 IRS 5. & 6.7 & $6.00 \pm 0.25$ & $9.80 \pm 0.33$ & $19.4 \pm 0.29$ & & $104.7 \pm 0.79$ & $17.4 \pm 0.54$ & $68.0 \pm 0.79$ \\
\hline L1535 IRS . & 5.5 & $2.80 \pm 0.14$ & $2.23 \pm 0.16$ & $8.5 \pm 0.22$ & $26.2 \pm 1.9$ & $15.9 \pm 0.34$ & $2.3 \pm 0.46$ & $19.1 \pm 0.48$ \\
\hline TMR $1 \ldots \ldots$ & 6.2 & $4.10 \pm 0.18$ & $4.06 \pm 0.16$ & $11.6 \pm 0.23$ & $23.6 \pm 1.8$ & $40.3 \pm 0.46$ & $8.6 \pm 0.61$ & $51.4 \pm 0.45$ \\
\hline TMC 1A. & 6.6 & $1.31 \pm 0.17$ & $2.83 \pm 0.19$ & $6.6 \pm 0.24$ & $25.3 \pm 1.4$ & $34.6 \pm 0.60$ & $18.4 \pm 0.80$ & $28.8 \pm 0.42$ \\
\hline L1527 IRS . & 5.9 & $2.97 \pm 0.26$ & $2.81 \pm 0.18$ & $10.6 \pm 0.35$ & $24.2 \pm 0.4^{\mathrm{a}}$ & $51.3 \pm 0.61$ & $4.1 \pm 0.31$ & $50.9 \pm 0.75$ \\
\hline TMC $1 \ldots \ldots \ldots \ldots$ & 5.2 & $2.32 \pm 0.20$ & $4.41 \pm 0.21$ & $4.07 \pm 0.27$ & $21.3 \pm 2.5$ & $16.0 \pm 0.59$ & $7.1 \pm 0.45$ & $21.3 \pm 0.43$ \\
\hline
\end{tabular}

a JCMT Archive data, previously presented by MacLeod et al. 1994. 

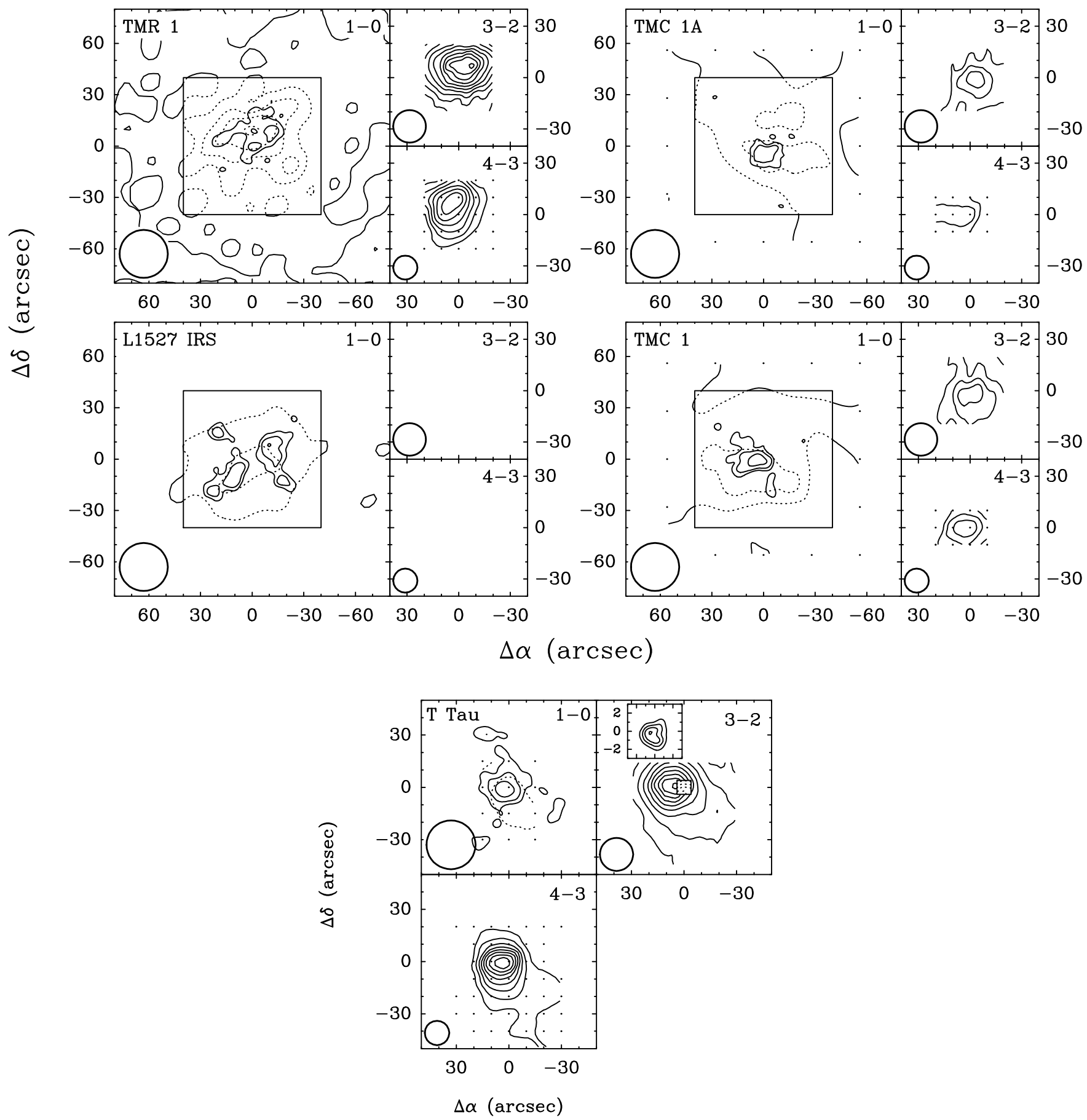

FIG. 3.-Continued

narrow absorption features. Since care was taken to obtain emission-free reference positions for the position-switched observations, these features are interpreted as selfabsorption. Two-thirds of the sources have higher intensities on the blue side of the absorption features in ${ }^{12} \mathrm{CO}$ $4-3$ and $6-5$, while no preferentially red or blue asymmetry is seen in ${ }^{12} \mathrm{CO} 3-2$. The presence of redshifted absorption is interpreted as evidence for infall (cf. Gregersen et al. 1997; Mardones et al. 1997), although detailed modeling of the line profiles, including contributions from the outflow, is required for any definitive conclusion to be reached.

Estimates of the opacity at line center $\left(\tau_{\max }\right)$ and averaged over the line profile $(\bar{\tau})$ are obtained from the ${ }^{13} \mathrm{CO} / \mathrm{C}^{18} \mathrm{O}$
3-2 and ${ }^{12} \mathrm{CO} /{ }^{13} \mathrm{CO} 6-5$ ratios. The opacity in the outflow, $\tau_{\text {wing }}$, is obtained from the ${ }^{12} \mathrm{CO} /{ }^{13} \mathrm{CO} 3-2$ ratios averaged over the line wings. Again, the standard abundances of $\left[{ }^{12} \mathrm{CO}\right]:\left[{ }^{13} \mathrm{CO}\right]=65: 1$ and $\left[{ }^{13} \mathrm{CO}\right]:\left[\mathrm{C}^{18} \mathrm{O}\right]=8: 1$ are adopted (Table 6). Typical ${ }^{13} \mathrm{CO} 3-2$ opacities of $\bar{\tau}(13)=$ 0.5-5.6 are found, with the exception of the deeply embedded source TMC 1 , where even $\mathrm{C}^{18} \mathrm{O} 3-2$ is optically thick, with $\bar{\tau}(18)>3$. For ${ }^{12} \mathrm{CO} 6-5$, optical depths of $\bar{\tau}(12)=$ 5-67 are found, indicating optically thin emission in ${ }^{13} \mathrm{CO}$ 6-5 toward all sources. The maximum opacities at 3-2 and 6-5 are much larger than the line-averaged values, but only at the few velocity channels covering the self-absorption features. Line wing ${ }^{12} \mathrm{CO} 3-2$ opacities, $\tau_{\text {wing }}(12)$, are gener- 


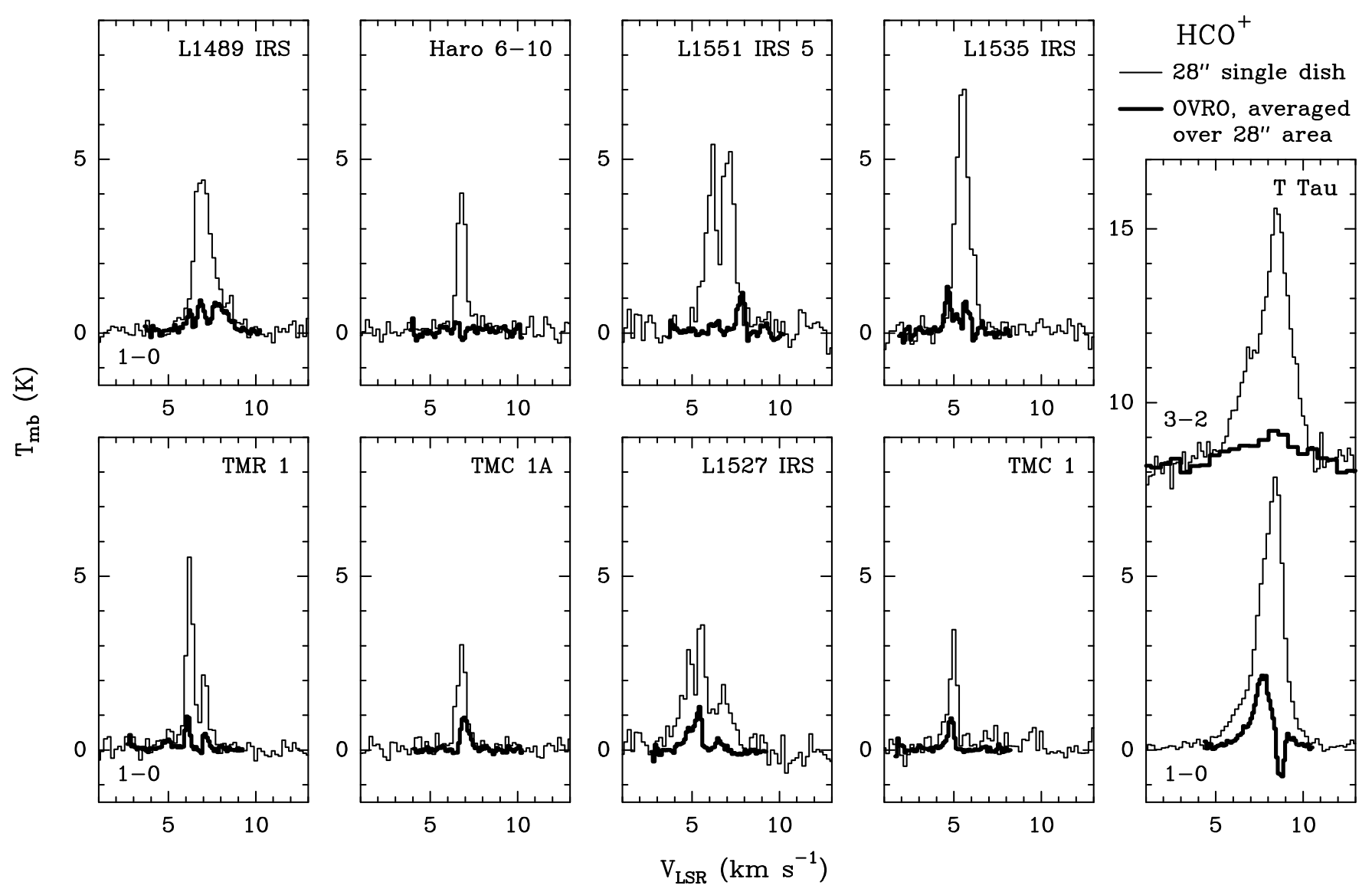

FIG. 4. - $\mathrm{HCO}^{+} 1-0$ spectra observed in the $28^{\prime \prime}$ IRAM $30 \mathrm{~m}$ beam (thin lines), overlaid with the OVRO spectra, after convolution with a $28^{\prime \prime}$ beam (heavy lines). All spectra are on the same intensity scale.
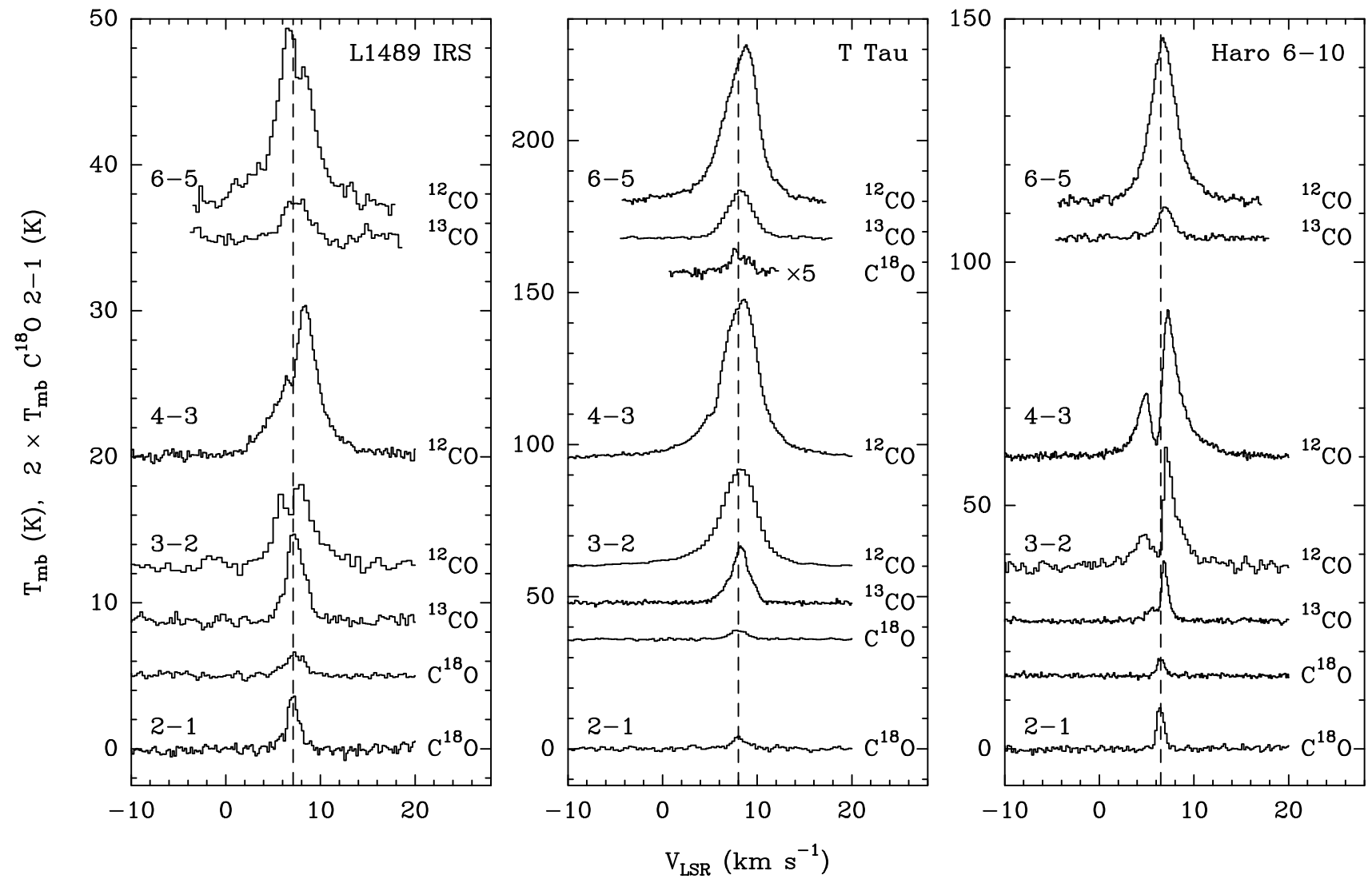

FIG. 5.- Single-dish ${ }^{12} \mathrm{CO},{ }^{13} \mathrm{CO}$, and $\mathrm{C}^{18} \mathrm{O}$ spectra. The $\mathrm{C}^{18} \mathrm{O} 2-1$ spectra have been multiplied by 2 for clarity. The vertical dashed lines indicate the systemic velocity of the objects. 

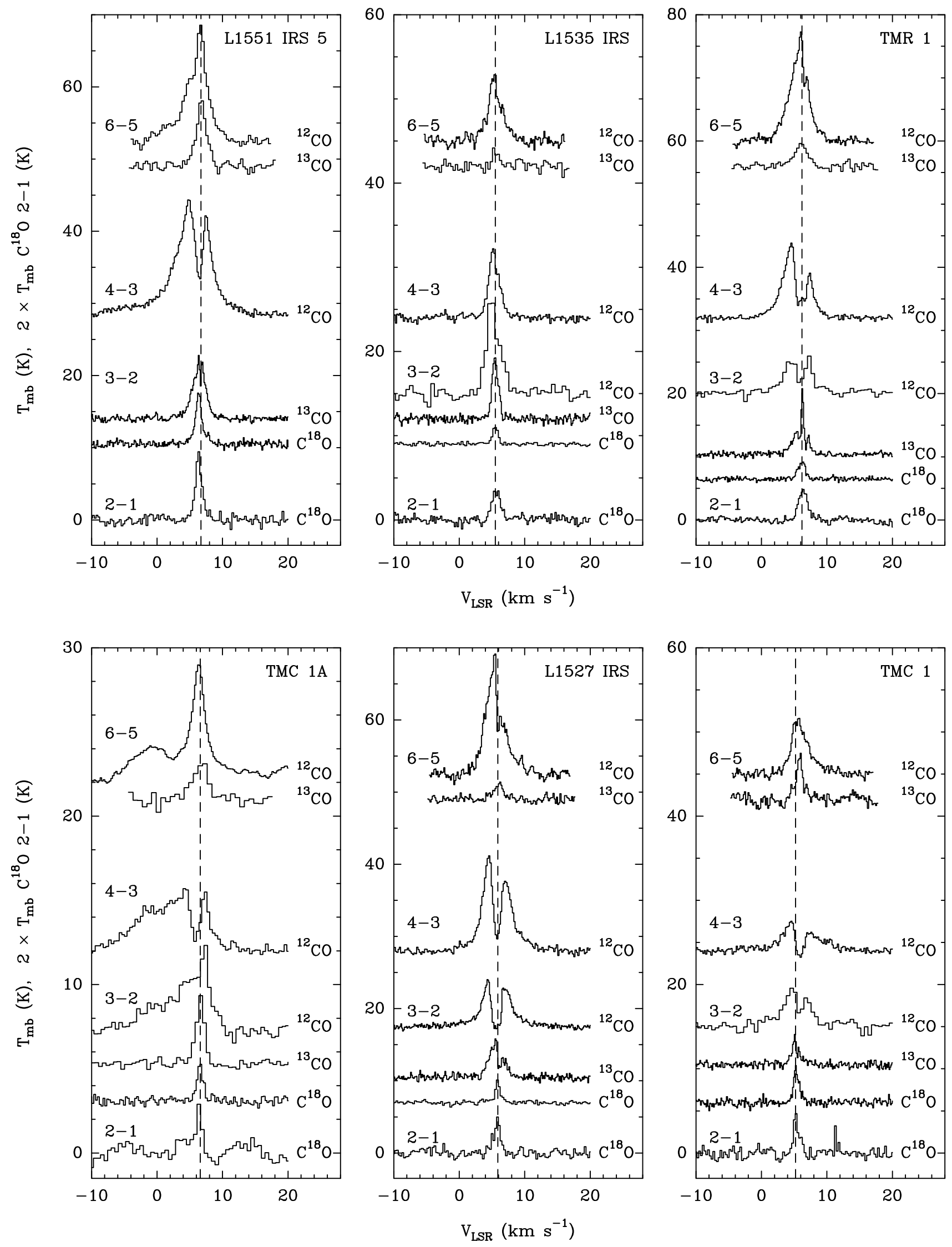

FIG. 5.-Continued 
TABLE 6

Single-Dish CO Line OpaCities

\begin{tabular}{|c|c|c|c|c|c|c|c|}
\hline \multirow[b]{2}{*}{ SOURCE } & \multicolumn{4}{|c|}{${ }^{13} \mathrm{CO} / \mathrm{C}^{18} \mathrm{O} 3-2$} & \multicolumn{2}{|c|}{${ }^{12} \mathrm{CO} /{ }^{13} \mathrm{CO} 3-2$} & \multirow{2}{*}{$\frac{{ }^{12} \mathrm{CO} /{ }^{13} \mathrm{CO} 6-5}{\bar{\tau}(12)}$} \\
\hline & $\tau_{\max }(13)$ & $\tau_{\max }(12)$ & $\bar{\tau}(13)$ & $\bar{\tau}(12)$ & $\tau_{\text {wing }}(12)$ & $\tau_{\max }(12)$ & \\
\hline L1489 IRS . & 3.5 & 230 & 2.3 & 150 & 8.3 & 40 & 16 \\
\hline T Tau $\ldots \ldots \ldots \ldots$ & 1.5 & 100 & 0.5 & 33 & 4.9 & 30 & 16 \\
\hline Haro $6-10 \ldots . .$. & 25.0 & 1625 & 1.8 & 117 & 4.2 & 15 & 8 \\
\hline L1551 IRS $5 . . .$. & 18.5 & 1200 & 5.6 & 364 & $\ldots$ & 60 & 19 \\
\hline L1535 IRS ........ & 2.7 & 175 & 1.1 & 72 & $<8.4$ & 24 & 8 \\
\hline TMR $1 \ldots \ldots \ldots \ldots$ & 7.5 & 490 & 3.3 & 215 & 8.9 & 22 & 12 \\
\hline TMC $1 \mathrm{~A} \ldots . . . .$. & 6.5 & 425 & 4.5 & 290 & $<0.5$ & 100 & 67 \\
\hline L1527 IRS ........ & $>24$ & $>1560$ & 2.1 & 137 & 18.4 & 18 & 5 \\
\hline TMC $1 \ldots \ldots \ldots \ldots$ & $>24$ & $>1560$ & $>24$ & $>1560$ & $<3.3$ & 110 & 26 \\
\hline
\end{tabular}

ally an order of magnitude lower than the value obtained over the full line profile, with the only exceptions of TMC $1 \mathrm{~A}$ and TMC 1, where the wings are found to be optically thin.

Constraints on the excitation of the line cores and line wings are obtained from the ratio of the ${ }^{13} \mathrm{CO} 3-2$ and 6-5 transitions, and the ${ }^{12} \mathrm{CO} 4-3$ and 6-5 lines, respectively. Calculations are made using an escape probability formalism under optically thin conditions, with the collision rates from Flower \& Launay (1985) and Schinke et al. (1985). Neglecting the moderate optical depths of the ${ }^{13} \mathrm{CO} 3-2$ line cores and of the ${ }^{12} \mathrm{CO} 4-3$ and 6-5 line wings, the observed line ratios constrain the excitation, $n_{\mathrm{H}_{2}}$ and $T_{\text {kin }}$. The observed line ratios are listed in Table 7, together with the inferred kinetic temperatures assuming thermalization, i.e., $n_{\mathrm{H}_{2}}>10^{5} \mathrm{~cm}^{-3}$. If the actual density is smaller or the line opacity cannot be neglected, the inferred kinetic temperatures are increased or decreased, respectively. For the material at line center, the temperature is constrained to 35-55 K for L1489 IRS, Haro 6-10, TMR 1, and TMC 1A. For T Tau, L1551 IRS 5, and TMC 1 the temperature range may extend up to $120 \mathrm{~K}$, because of the uncertainty in the line ratio. The extreme line ratios of 3.5-4.5 observed toward L1535 IRS and L1527 IRS limit the temperature to $\sim 25 \mathrm{~K}$. All these temperatures agree within the accuracy to the dust temperatures inferred by Moriarty-Schieven et al. (1995). The range of temperatures found for the line wings is 25-70 K for most sources, similar to the range of temperatures for the cores. Only for L1535 IRS and L1527 IRS are the inferred values of $70 \mathrm{~K}$ and $45 \mathrm{~K}$, respectively, for the wings significantly larger than those for the core, $\sim 25$ $\mathrm{K}$. In the determination of the outflow mass below, an exci- tation temperature of $T_{\mathrm{ex}}=50 \mathrm{~K}$ will be assumed for the line wings, based on the observed ratios.

\subsection{Outflow Maps}

Outflow maps in ${ }^{12} \mathrm{CO} 3-2$ are presented in Figure 6. The maps have a resolution of $15^{\prime \prime}$, except for T Tau, which has been mapped with the $21^{\prime \prime}$ CSO beam. Table 8 summarizes the maximum velocity extent of the red and blue emission, determined from the ${ }^{12} \mathrm{CO} 4-3$ spectra, which offer the best signal-to-noise ratio on the line wings. The velocities at which the emission is blended with the surrounding cloud are determined from the ${ }^{12} \mathrm{CO} 3-2$ position-velocity diagrams of Figure 7, and have been excluded from the outflow emission. For T Tau, no clear position-velocity correlation was observed, and a range of $6.0-8.0 \mathrm{~km} \mathrm{~s}^{-1}$ was excluded. The close orientation to the plane of the sky of the L1527 IRS outflow prevents unambiguous separation of outflow and core emission; excluding the velocity range of 5.0-7.0 $\mathrm{km} \mathrm{s}^{-1}$ is found to give the clearest outflow map.

Outflow emission is associated with all sources, but a clear bipolar morphology is only seen toward L1489 IRS, TMC 1A, TMC 1, and L1527 IRS, as shown previously for the latter three sources by Chandler et al. (1996) and MacLeod et al. (1994). Toward TMR 1, the red and blue emission also overlaps, but the overall structure is consistent with the previous interferometric results of Terebey et al. (1990). In spite of pronounced line wings, no clear bipolar structure is seen toward Haro 6-10. Toward L1535 IRS, line wings are only prominent in the ${ }^{12} \mathrm{CO} 6-5$ spectra, and the source is therefore classified as an outflow source. In ${ }^{12} \mathrm{CO} 3-2$, the outflow emission is almost undetected, and its structure is undetermined. The outflow of T Tau is

TABLE 7

Single-Dish CO Line Excitation

\begin{tabular}{|c|c|c|c|c|c|}
\hline \multirow[b]{2}{*}{ SOURCE } & \multicolumn{2}{|c|}{ LINE CORE } & \multicolumn{2}{|c|}{ LINE WINGS } & \multirow[b]{2}{*}{$\begin{array}{c}T_{\text {dust }}{ }^{\mathrm{a}} \\
(\mathbf{K})\end{array}$} \\
\hline & ${ }^{13} \mathrm{CO}^{(3-2) /(6-5)}$ & $\begin{array}{l}T_{\text {kin }} \\
(\mathrm{K})\end{array}$ & ${ }^{12} \mathrm{CO}^{(4-3) /(6-5)}$ & $\begin{array}{l}T_{\text {kin }} \\
(\mathbf{K})\end{array}$ & \\
\hline L1489 IRS . & $1.0-2.0$ & $38-55$ & $1.0-2.0$ & $35-70$ & 43 \\
\hline T Tau $\ldots \ldots \ldots \ldots$ & $0.5-1.0$ & $55-120$ & $1.5-3.0$ & $24-45$ & 51 \\
\hline Haro $6-10 \ldots \ldots \ldots$ & $1.0-2.0$ & $38-55$ & $1.0-2.0$ & $35-70$ & \\
\hline L1551 IRS $5 \ldots \ldots$ & $0.5-2.0$ & $38-120$ & $1.5-4.0$ & $25-45$ & 47 \\
\hline L1535 IRS ........ & $3.5-4.5$ & 25 & 1.0 & 70 & 40 \\
\hline TMR $1 \ldots \ldots \ldots \ldots$ & $1.0-1.8$ & $40-55$ & $1.0-2.0$ & $35-70$ & 53 \\
\hline TMC $1 \mathrm{~A} \ldots \ldots \ldots$ & $1.0-2.0$ & $38-55$ & $1.5-3.5$ & $25-45$ & 44 \\
\hline L1527 IRS ........ & $3.5-4.5$ & 25 & 1.5 & 45 & 31 \\
\hline TMC $1 \ldots \ldots \ldots \ldots$ & $0.5-2.0$ & $38-120$ & $1.0-2.0$ & $35-70$ & 41 \\
\hline
\end{tabular}

${ }^{\text {a }}$ From Moriarty-Schieven et al. 1995. 

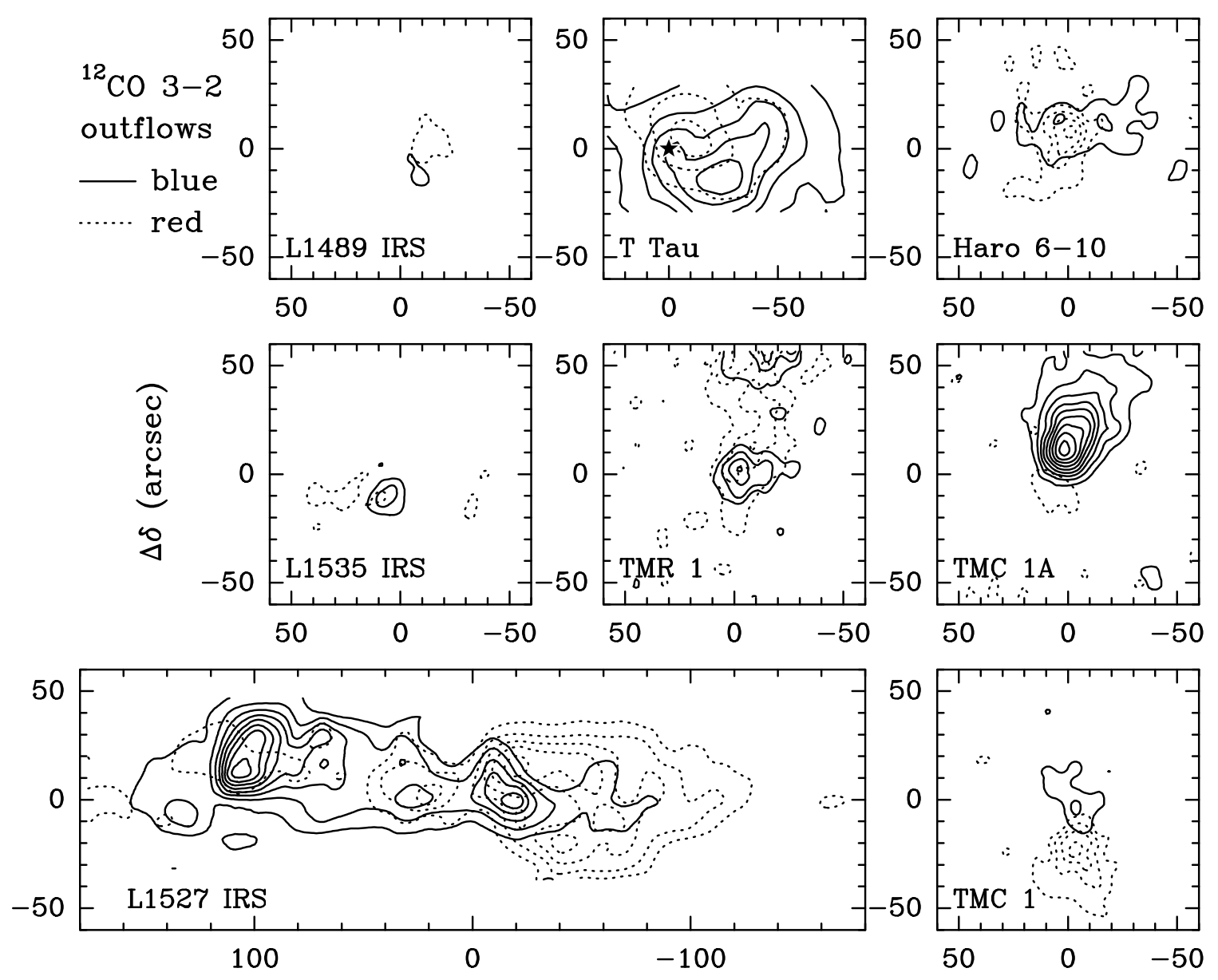

$\Delta \alpha(\operatorname{arcsec})$

FIG. 6.-JCMT ${ }^{12} \mathrm{CO} 3-2$ outflow maps. Solid lines show blueshifted emission, dashed lines show redshifted emission. Contours are drawn at the $3 \sigma$ level. Note that the map of T Tau is not centered on the position of the source, which is indicated by a star. The map of L1527 IRS has been presented previously by MacLeod et al. (1994).

known to be close to pole-on, resulting in superposed red and blue lobes (Herbst, Koresko, \& Leinert 1995; van Langevelde et al. 1994b; Schuster et al. 1997).

Except for Haro 6-10 and L1535 IRS, position angles for the outflows can be derived from the maps, and are listed in Table 8. For Haro 6-10, a position angle of $65^{\circ}$ is adopted from the detection of Herbig-Haro objects in that direction (Strom et al. 1986). For L1535 IRS, a position angle of $10^{\circ}$ is inferred from a $K^{\prime}$ scattered-light image presented by Hodapp (1994; see Fig. 10). Values for T Tau $\left(160^{\circ}\right)$ and L1551 IRS $5\left(45^{\circ}\right)$ are taken from van Langevelde et al. (1994b) and Moriarty-Schieven \& Snell (1988). Because of its pole-on configuration, attributing a position angle to the outflow of T Tau is not very meaningful.

The extent of the outflow emission of L1489 IRS, Haro 6-10, L1535 IRS, TMC 1A, and TMC 1 is $\lesssim 1^{\prime}$ (Figs. 6 and 7, Table 8), in marked contrast to the outflows of L1527 IRS $\left(R \approx 3^{\prime}\right)$ and L1551 IRS $5\left(R \approx 10^{\prime}\right)$. Due to the orientation of T Tau, the projected size of its outflow $\left(\sim 1^{\prime}\right)$ could be much less than its true extent. Although the orientation of the position-velocity diagrams of TMR 1 and Haro 6-10 deviate from the outflow directions of Table 8 by $5^{\circ}$ and $15^{\circ}$, respectively, it seems unlikely that significant outflow emission was missed.
The mass contained in the outflow lobes is listed in Table 8 , using equation (1) with main-beam antenna temperature $T_{\mathrm{mb}}$ instead of brightness temperature $T_{b}$, which reduces to $M / M_{\odot}=1.46 \times 10^{-6} \int T_{\text {mb }}\left[\tau_{\text {wing }} /\left(1-e^{-\tau_{\text {wing }}}\right)\right] d V$ for $T_{\text {ex }}=50 \mathrm{~K}$. Only pixels with signal $\geq 3 \sigma$ are included in the estimates. Values for $\tau_{\text {wing }}$ are taken from Table 6. For L1551 IRS 5, the mass from Moriarty-Schieven \& Snell (1988) is listed, which is already corrected for opacity. The inferred outflow masses are typically $0.5 \%-1.5 \%$ of the mass of the surrounding cloud, as inferred from the line core intensity over the full mapped areas of $2^{\prime} \times 2^{\prime}$. Toward the extremely weak outflow source L1535 IRS, the lobes carry no more than $0.1 \%$ of the mass at line center, or $3.5 \times 10^{-4}$ $M_{\odot}$. In contrast, the prominent outflows of L1551 IRS 5 (Moriarty-Schieven \& Snell 1988), L1527 IRS, and T Tau contain as much as $\sim 7 \%$ of the total mass at line center, with $3.1,0.18$, and $0.033 M_{\odot}$, respectively. These masses are not corrected for inclination, which can project a significant fraction of the outflowing mass to velocities close to systemic (cf. masses listed in Table 8).

Various methods exist to derive flow parameters corrected for inclination from the measured, projected maximum outflow velocity, spatial extent, and mass. Cabrit $\&$ Bertout (1990) have derived correction factors for the 
TABLE 8

${ }^{12} \mathrm{CO} 3-2$ Outflow Parameters

\begin{tabular}{|c|c|c|c|c|c|c|c|c|c|}
\hline Source & $\begin{array}{c}i \\
(\operatorname{deg})\end{array}$ & $\begin{array}{l}\text { P.A. } \\
\text { (deg) }\end{array}$ & $\begin{array}{c}V_{\max }^{\mathrm{a}} \\
\left(\mathrm{km} \mathrm{s}^{-1}\right)\end{array}$ & $\begin{array}{l}R^{\mathrm{a}} \\
(\mathrm{AU})\end{array}$ & $\begin{array}{l}M^{\mathrm{b}, \mathrm{g}} \\
\left(M_{\odot}\right)\end{array}$ & $\begin{array}{l}t_{d}^{\mathrm{a}, \mathrm{c}} \\
(\mathrm{yr})\end{array}$ & $\begin{array}{c}\dot{M}^{\mathrm{a}, \mathrm{d}} \\
\left(M_{\odot} \mathrm{yr}^{-1}\right)\end{array}$ & $\begin{array}{c}F_{\mathrm{CO}}^{\mathrm{e}, \mathrm{g}} \\
\left(M_{\odot} \mathrm{km} \mathrm{s}^{-1} \mathrm{yr}^{-1}\right)\end{array}$ & $\begin{array}{c}L_{\mathrm{kin}}^{\mathrm{f}, \mathrm{g}} \\
\left(L_{\odot}\right)\end{array}$ \\
\hline \multicolumn{10}{|c|}{ Blue lobe } \\
\hline L1489 IRS . & 60 & 165 & 8.1 & $4.2 \mathrm{E} 3$ & $6.6 \mathrm{E}-4$ & $2.5 \mathrm{E}+3$ & $2.9 \mathrm{E}-7$ & $2.5 \mathrm{E}-6$ & $2.3 \mathrm{E}-3$ \\
\hline Т Tau $\ldots \ldots \ldots \ldots$ & 15 & 160 & 15.6 & $8.4 \mathrm{E} 3$ & $1.6 \mathrm{E}-2$ & $2.7 \mathrm{E}+3$ & $6.4 \mathrm{E}-6$ & $4.0 \mathrm{E}-5$ & $4.3 \mathrm{E}-2$ \\
\hline Haro 6-10. & 30 & 65 & 12.0 & $5.6 \mathrm{E} 3$ & $1.0 \mathrm{E}-3$ & $2.2 \mathrm{E}+3$ & $4.4 \mathrm{E}-7$ & $3.2 \mathrm{E}-6$ & $3.3 \mathrm{E}-3$ \\
\hline L1551 IRS $5 \ldots \ldots$ & 65 & 45 & 19.7 & $1.6 \mathrm{E} 5$ & $1.6 \mathrm{E} 0$ & $3.8 \mathrm{E}+4$ & $4.8 \mathrm{E}-5$ & $9.9 \mathrm{E}-4$ & $2.3 \mathrm{E} 0$ \\
\hline L1535 IRS ......... & 60 & 10 & 3.0 & $3.5 \mathrm{E} 3$ & $9.1 \mathrm{E}-5$ & $5.5 \mathrm{E}+3$ & $1.9 \mathrm{E}-8$ & $6.0 \mathrm{E}-8$ & $2.0 \mathrm{E}-5$ \\
\hline TMR $1 \ldots \ldots \ldots \ldots$ & 60 & 170 & 8.7 & $7.0 \mathrm{E} 3$ & $8.9 \mathrm{E}-4$ & $3.8 \mathrm{E}+3$ & $2.8 \mathrm{E}-7$ & $2.4 \mathrm{E}-6$ & $2.5 \mathrm{E}-3$ \\
\hline TMC $1 \mathrm{~A} \ldots \ldots \ldots$ & 55 & 155 & 16.6 & $8.4 \mathrm{E} 3$ & $1.6 \mathrm{E}-3$ & $2.4 \mathrm{E}+3$ & $8.0 \mathrm{E}-7$ & $8.0 \mathrm{E}-6$ & $1.3 \mathrm{E}-2$ \\
\hline L1527 IRS ........ & 75 & 90 & 6.9 & $2.2 \mathrm{E} 4$ & $7.7 \mathrm{E}-2$ & $1.5 \mathrm{E}+4$ & $5.7 \mathrm{E}-6$ & $4.1 \mathrm{E}-5$ & $3.3 \mathrm{E}-2$ \\
\hline TMC $1 \ldots \ldots \ldots \ldots$ & 55 & 0 & 10.7 & $6.3 \mathrm{E} 3$ & $1.3 \mathrm{E}-4$ & $2.8 \mathrm{E}+3$ & $5.3 \mathrm{E}-8$ & $3.4 \mathrm{E}-7$ & $9.6 \mathrm{E}-4$ \\
\hline \multicolumn{10}{|c|}{ Red lobe } \\
\hline L1489 IRS ........ & 60 & 165 & 7.4 & $2.1 \mathrm{E} 3$ & $1.6 \mathrm{E}-3$ & $1.3 \mathrm{E}+3$ & $1.4 \mathrm{E}-6$ & $1.1 \mathrm{E}-5$ & $9.1 \mathrm{E}-3$ \\
\hline T Tau .............. & 15 & 160 & 12.0 & $7.0 \mathrm{E} 3$ & $1.7 \mathrm{E}-2$ & $2.8 \mathrm{E}+3$ & $6.9 \mathrm{E}-6$ & $3.3 \mathrm{E}-5$ & $2.9 \mathrm{E}-2$ \\
\hline Haro $6-10 \ldots \ldots \ldots$ & 30 & 65 & 13.0 & $4.2 \mathrm{E} 3$ & $1.3 \mathrm{E}-3$ & $1.5 \mathrm{E}+3$ & $7.9 \mathrm{E}-7$ & $6.2 \mathrm{E}-6$ & $6.6 \mathrm{E}-3$ \\
\hline L1551 IRS $5 \ldots \ldots$ & 65 & 45 & 15.8 & $7.6 \mathrm{E} 4$ & $1.5 \mathrm{E} 0$ & $2.3 \mathrm{E}+4$ & $7.4 \mathrm{E}-5$ & $1.2 \mathrm{E}-3$ & $2.3 \mathrm{E} 0$ \\
\hline L1535 IRS ........ & 60 & 10 & 5.0 & $2.8 \mathrm{E} 3$ & $6.0 \mathrm{E}-5$ & $2.7 E+3$ & $2.6 \mathrm{E}-8$ & $1.3 \mathrm{E}-7$ & $7.7 \mathrm{E}-5$ \\
\hline TMR $1 \ldots \ldots \ldots \ldots$ & 60 & 170 & 4.3 & $5.6 \mathrm{E} 3$ & $1.5 \mathrm{E}-3$ & $6.2 E+3$ & $2.9 \mathrm{E}-7$ & $1.3 \mathrm{E}-6$ & $6.2 \mathrm{E}-4$ \\
\hline TMC $1 \mathrm{~A} \ldots \ldots \ldots$ & 55 & 155 & 6.5 & $2.8 \mathrm{E} 3$ & $3.2 \mathrm{E}-5$ & $2.0 \mathrm{E}+3$ & $1.8 \mathrm{E}-8$ & $7.1 \mathrm{E}-7$ & $4.6 \mathrm{E}-5$ \\
\hline L1527 IRS ............ & 75 & 90 & 9.6 & $1.8 \mathrm{E} 4$ & $9.9 \mathrm{E}-2$ & $9.0 \mathrm{E}+3$ & $1.2 \mathrm{E}-5$ & $1.2 \mathrm{E}-4$ & $1.3 \mathrm{E}-1$ \\
\hline TMC $1 \ldots \ldots \ldots . .$. & 55 & 0 & 9.3 & $4.2 \mathrm{E} 3$ & $7.1 \mathrm{E}-4$ & $2.1 E+3$ & $4.0 \mathrm{E}-7$ & $2.3 E-6$ & $2.0 \mathrm{E}-3$ \\
\hline
\end{tabular}

${ }^{\text {a }}$ Not corrected for inclination.

${ }^{\mathrm{b}}$ Assuming $T_{\mathrm{ex}}=60 \mathrm{~K}$ and line wings opacities $\tau_{\text {wing }}$ from Table 6 .

${ }^{c}$ Dynamical time $t_{d}=R / V_{\max }$.

d Mass outflow rate $\dot{M}=M / t_{d}$.

${ }^{\text {e }}$ Outflow force $F_{\mathrm{CO}}=M V_{\max }^{2} / R$.

${ }^{\mathrm{f}}$ Kinetic luminosity $L_{\mathrm{kin}}=\frac{1}{2} M V_{\mathrm{max}}^{3} / R$.

g Corrected for inclination, using average correction factors from Cabrit \& Bertout 1990.

mass $M$, the flow momentum rate, $F_{\mathrm{CO}}=M V_{\mathrm{max}}^{2} / R$, and the kinetic luminosity, $L_{\mathrm{kin}}=\frac{1}{2} M V_{\max }^{3} / R$, for three different realistic outflow models. We have used the average factor for these three models to correct the values of $M, F_{\mathrm{CO}}$, and $L_{\text {kin }}$. The spread between the different models is included in the error bars shown in Figure 8. Cabrit \& Bertout (1990) do not give correction factors for the dynamic time scale, $t_{d}=R / V$, or the mass outflow rate, $\dot{M}=M / t_{d}$.

The adopted inclinations, defined as the angle between the outflow direction and the line of sight, are based on the outflow morphology and near-infrared observations of scattered light (cf. Fig. 10). Kenyon et al. (1993) constrain $i$ to $60^{\circ}-90^{\circ}$ for L1489 IRS and L1535 IRS by modeling the near-infrared scattered-light images. The scattered-light images in $K^{\prime}$ (Hodapp 1994) also suggest that $i \approx 60^{\circ}$ is a good estimate for these sources. Herbst et al. $(1986,1997)$ find $i=13^{\circ}-19^{\circ}$ for $\mathrm{T}$ Tau. The lack of a bipolar morphology and the broad line wings suggests $i<45^{\circ}$ for Haro 6-10. For L1551 IRS 5, Moriarty-Schieven \& Snell (1988) quote $i \approx 65^{\circ}$. For TMR, $1 i \approx 60^{\circ}$ is inferred from the OVRO $\mathrm{HCO}^{+}$and ${ }^{13} \mathrm{CO}$ data and near-infrared observations by Terebey et al. (1990) (see $\S$ 5.1.6). Chandler et al. (1996) limit $i$ to $40^{\circ}-70^{\circ}$ for TMC $1 \mathrm{~A}$ and TMC 1 . The partial superposition of red and blue emission of L1527 IRS suggests $i>65^{\circ}$. The inferred inclinations are in good agreement with the scattered-light modeling results of Whitney, Kenyon, \& Gómez (1997).

The inclination-corrected flow parameters are listed in Table 8, and agree to within a factor of a few with the previous results of Cabrit \& Bertout (1992; hereafter CB92) for L1551 IRS 5 and with those of Chandler et al. (1996) for TMC 1A and TMC 1. The inferred outflow mass, and hence the flow force and kinetic luminosity, for T Tau is lower by a factor of 20 compared to the value cited by CB92, who used the results of Edwards \& Snell (1982), which were obtained with a much larger beam size of $1^{\prime}$ and may suffer from confusion with ambient cloud emission.

\subsection{The Relation between Outflow Force and Envelope Mass}

In Figure 8, the outflow momentum rate, or flow force, $F_{\mathrm{CO}}$, of the red and blue lobes is plotted against a number of source properties: bolometric luminosity, $1.1 \mathrm{~mm}$ continuum envelope flux, $2.7 \mathrm{~mm}$ continuum disk flux, and relative age as traced by $\int T_{\mathrm{mb}}\left(\mathrm{HCO}^{+} 3-2\right) d V / L_{\mathrm{bol}}$. For reference, the data points of CB92 and their derived $F_{\mathrm{CO}}-L_{\mathrm{bol}}$ relationship are also shown. Single-dish $1 \mathrm{~mm}$ fluxes of the CB92 sources have been taken from Terebey, Chandler, \& André (1993), Minchin, Ward-Thompson, \& White (1995), and Saraceno et al. (1996). Our data are in excellent agreement with the trend observed for comparable YSO samples of $F_{\mathrm{CO}}$ versus $L_{\mathrm{bol}}(\mathrm{CB} 92)$, and of $F_{\mathrm{CO}}$ versus 1 mm continuum flux (Saraceno et al. 1996; Bontemps et al. 1996). As noted by Saraceno et al. (1996), the correlation between $F_{\mathrm{CO}}$ and $1 \mathrm{~mm}$ flux is better than with $L_{\mathrm{bol}}$. The slight scatter of the CB92 sources toward larger $1 \mathrm{~mm}$ fluxes can be explained by the fact these contain an unknown contribution from circumstellar disks, typically $30 \%-75 \%$ in a 19" beam (Paper I), for which our data have been corrected. This illustrates the importance of obtaining spatially resolved information on the dust emission of embedded sources.

CB92 show that the $6 \mathrm{~cm}$ radio flux, which probably traces the ionized component of the wind driving the molecular outflow, is well correlated with the outflow force as inferred from the swept-up CO. This indicates that, at least 


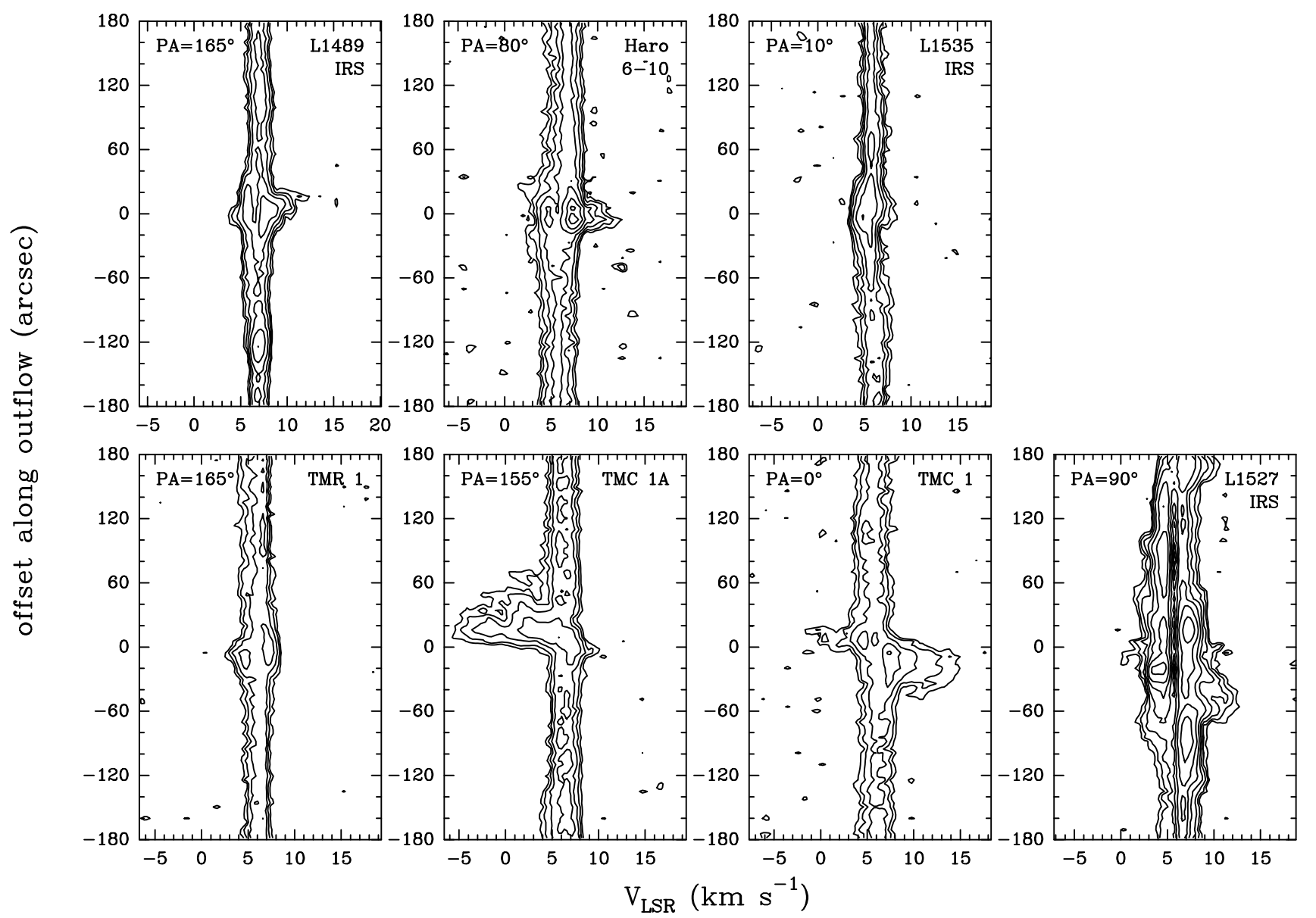

FIG. 7.-Position-velocity diagrams of ${ }^{12} \mathrm{CO} 3-2$ emission obtained with the JCMT on 6 ' long strips along the inferred outflow directions, as indicated. Contours are drawn at $3 \sigma$ intervals.

for embedded sources, $F_{\mathrm{CO}}$ is a measure of the intrinsic strength of the outflow, rather than simply reflecting the amount of $\mathrm{CO}$ available to be swept up. Bontemps et al. (1996) and Saraceno et al. (1996) propose that the relation between $1 \mathrm{~mm}$ flux and outflow strength can be explained by an increased mass infall rate in more massive envelopes. Our data allow the connection between envelope mass and outflow strength to be followed down to the scale of the accretion disk. In Paper I it was found that the 3.4 and 2.7 $\mathrm{mm}$ disk flux correlates with the envelope mass and that, as a result, disk flux is related to $F_{\mathrm{CO}}$. This supports the interpretation that more massive and denser envelopes have higher mass infall rates, possibly through variations in the sound speed (cf. Shu 1977). In that case, a higher disk mass is expected, as well as an increased accretion rate through the disk, resulting in a higher temperature and enhanced flux. The bolometric luminosity, thought to be dominated by accretion luminosity for embedded sources, also depends on the stellar mass and the viewing angle (cf. Yorke, Bodenheimer, \& Laughlin 1995), explaining its less tight relationship with $F_{\text {Co }}$.

The relative age of the object does not appear to be a good predictor of outflow strength. In Paper I it was argued that the ratio $\int T_{\mathrm{mb}}\left(\mathrm{HCO}^{+} 3-2\right) d V / L_{\mathrm{bol}}$ is a reliable measure of relative age and reflects the current ratio of envelope to stellar mass, with large values for young objects and low values for more evolved ones. No correlation with $F_{\mathrm{CO}}$ is found. Over a larger time span, extending from the class 0 to the $\mathrm{T}$ Tauri stage, age is expected to determine outflow strength, but within the embedded phase the envelope mass and infall rate are shown to dominate.

\section{SMALL-SCALE (700 AU) ENVELOPE STRUCTURE}

In this section, the compact structure traced by the $\mathrm{HCO}^{+},{ }^{13} \mathrm{CO}$, and $\mathrm{C}^{18} \mathrm{O}$ OVRO observations will be discussed in greater detail. Results on the individual sources are discussed in $\S 5.1$, and compared to the ${ }^{12} \mathrm{CO} 3-2$ outflows in Figure 9 and $K^{\prime}$ scattered light from the outflow cavities in Figure 10 (cf. Tamura et al. 1991; Whitney et al. 1997; Lucas \& Roche 1997). In $\S 5.2$, a simple picture will be constructed to explain the compact emission around the individual sources, consisting of three components: a core surrounding the young star, condensations scattered throughout the envelope, and material within the outflow or along its cavity walls.

\subsection{Individual Sources}

\subsubsection{L1489 IRS}

The OVRO $\mathrm{HCO}^{+},{ }^{13} \mathrm{CO}$, and $\mathrm{C}^{18} \mathrm{O} 1-0$ emission traces a $12^{\prime \prime} \times 7^{\prime \prime}$ core, elongated perpendicular to the outflow (Fig. 9). In addition, ${ }^{13} \mathrm{CO}$ emission is coincident with the southern outflow lobe, and $\mathrm{HCO}^{+}$emission is extended along the western edge of the northern outflow lobe and traces the site where the outflow borders on more extended $\mathrm{HCO}^{+}$emission (cf. Fig. 3). This latter feature is most 

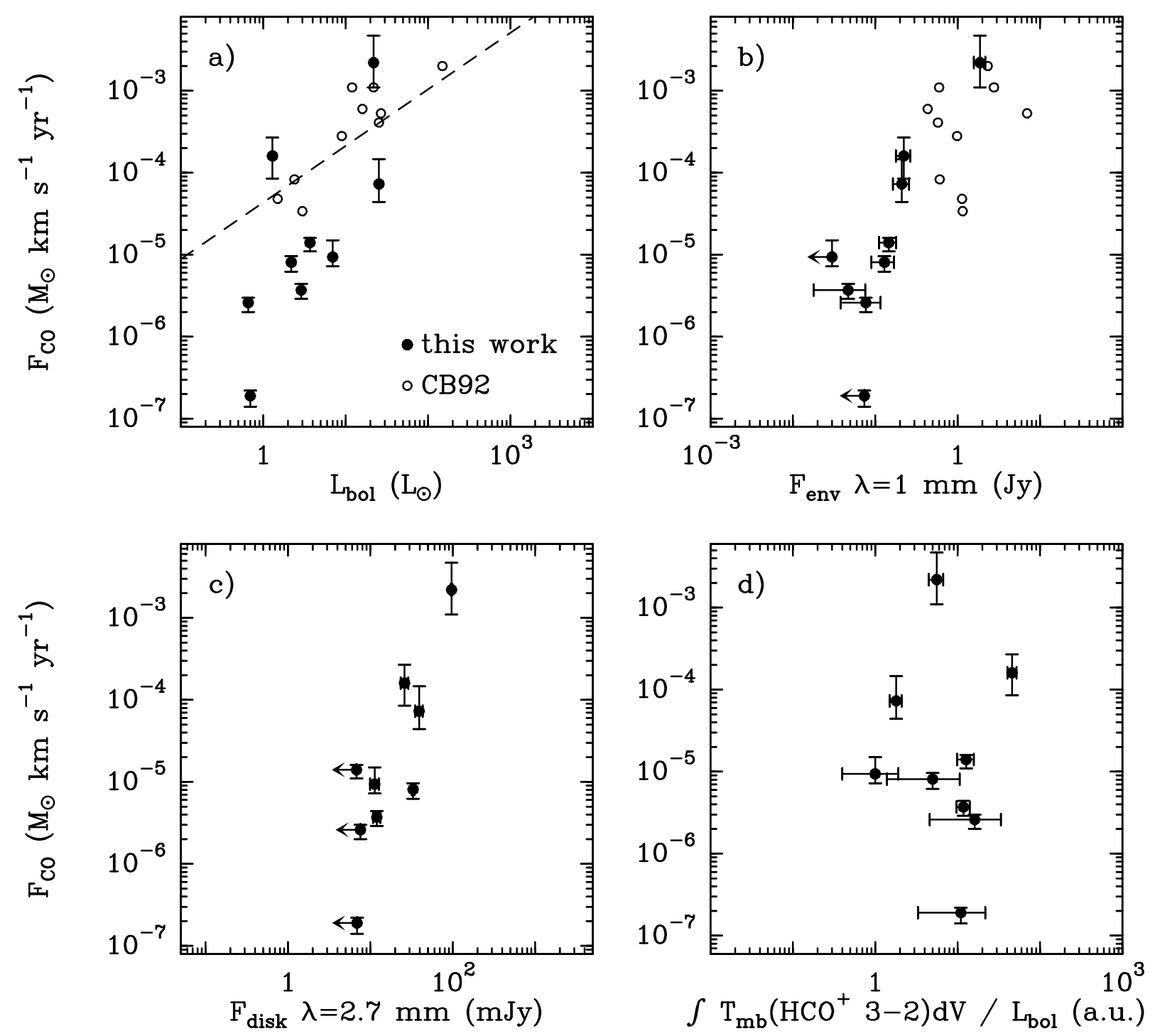

FIG. 8. - (a) Outflow force $F_{\text {CO }}=M V_{\text {max }}^{2} / R$ vs. bolometric luminosity ( filled symbols). The data points of CB92 are indicated by open symbols. (b) $F_{\text {Co }}$ vs. $1.1 \mathrm{~mm}$ envelope flux, from Paper I ( filled symbols). These fluxes have been corrected for the contribution of any circumstellar disk, and measure envelope mass alone. The data points of CB92 are again shown by open symbols. Note that their fluxes may still contain contributions from circumstellar disks, explaining the scatter toward higher values. $(c) F_{\mathrm{CO}}$ vs. $2.7 \mathrm{~mm}$ disk flux, from Paper I. $(d) F_{\mathrm{Co}}$ vs. relative age, as measured by the ratio of $\mathrm{HCO}^{+} 3-2$ intensity over $L_{\mathrm{bol}}$ (see Paper I). Young objects are to the right, older ones to the left of the plot. In all panels, $F_{\mathrm{CO}}$ has been corrected for line opacity and source inclination (see Table 8) using the correction factors determined by Cabrit \& Bertout (1990). The vertical error bars show the spread of these factors for different outflow models.

clearly seen in the zero-moment image of Figure 9, which better brings out low-level emission. $K^{\prime}$ scattered light is associated with the southern, blueshifted outflow lobe (Fig. 10); the extension to the southeast coincides with the lowest $\mathrm{HCO}^{+}$contour, and is probably part of the cavity wall. A total mass of $0.013 M_{\odot}$ is traced by the $\mathrm{C}^{18} \mathrm{O}$ emission over $20^{\prime \prime} \times 20^{\prime \prime}$, consistent with the dust mass of $0.016-0.025 M_{\odot}$ found in Paper I and the $0.04 M_{\odot}$ inferred from CS interferometry by Ohashi et al. (1996b).

The $\mathrm{HCO}^{+},{ }^{13} \mathrm{CO}$, and $\mathrm{C}^{18} \mathrm{O}$ OVRO spectra have a total width of $4 \mathrm{~km} \mathrm{~s}^{-1}$ (Fig. 2), and the $\mathrm{HCO}^{+}$position-velocity diagram obtained along the core's major axis could indicate rotation around a $0.9 M_{\odot}$ object, correcting for an inclination of $60^{\circ}$ (Fig. 11). This is larger than the $0.4 M_{\odot}$ inferred from $L_{\text {bol }}$ (Table 1), possibly because the $\mathrm{HCO}^{+}$contains contributions from the outflow, increasing the velocity width and overestimating the stellar mass. The deep absorption feature in $\mathrm{HCO}^{+}$at $+7.1 \mathrm{~km} \mathrm{~s}^{-1}$ corresponds to large-scale, optically thick material.

\subsection{2. $T$ Tau}

In $\mathrm{HCO}^{+},{ }^{13} \mathrm{CO}$, and $\mathrm{C}^{18} \mathrm{O} 1-0$, the emission reveals a marginally resolved core of $0.009 M_{\odot}$, or $30 \%$ of the mass inferred from the dust, a north-south ridge in $\mathrm{HCO}^{+}$and ${ }^{13} \mathrm{CO}$, and $\mathrm{HCO}^{+}$emission associated with the reflection nebula NGC 1555 (Figs. 1 and 10). Momose et al. (1996) interpret this structure seen in a larger ${ }^{13} \mathrm{CO}$ interferometer map as an the expanding shell around the pole-on outflow cavity. The peak of the central bright core is also picked up in $\mathrm{HCO}^{+} 3-2$ by OVRO (Hogerheijde et al. 1997b; reproduced here in Fig. 3c), and is interpreted as the bright cavity walls close to the base of the pole-on outflow.

The spectra seen in the OVRO beam have a FWHM of $\sim 3 \mathrm{~km} \mathrm{~s}^{-1}$ (Fig. 2), with a deep absorption feature in 

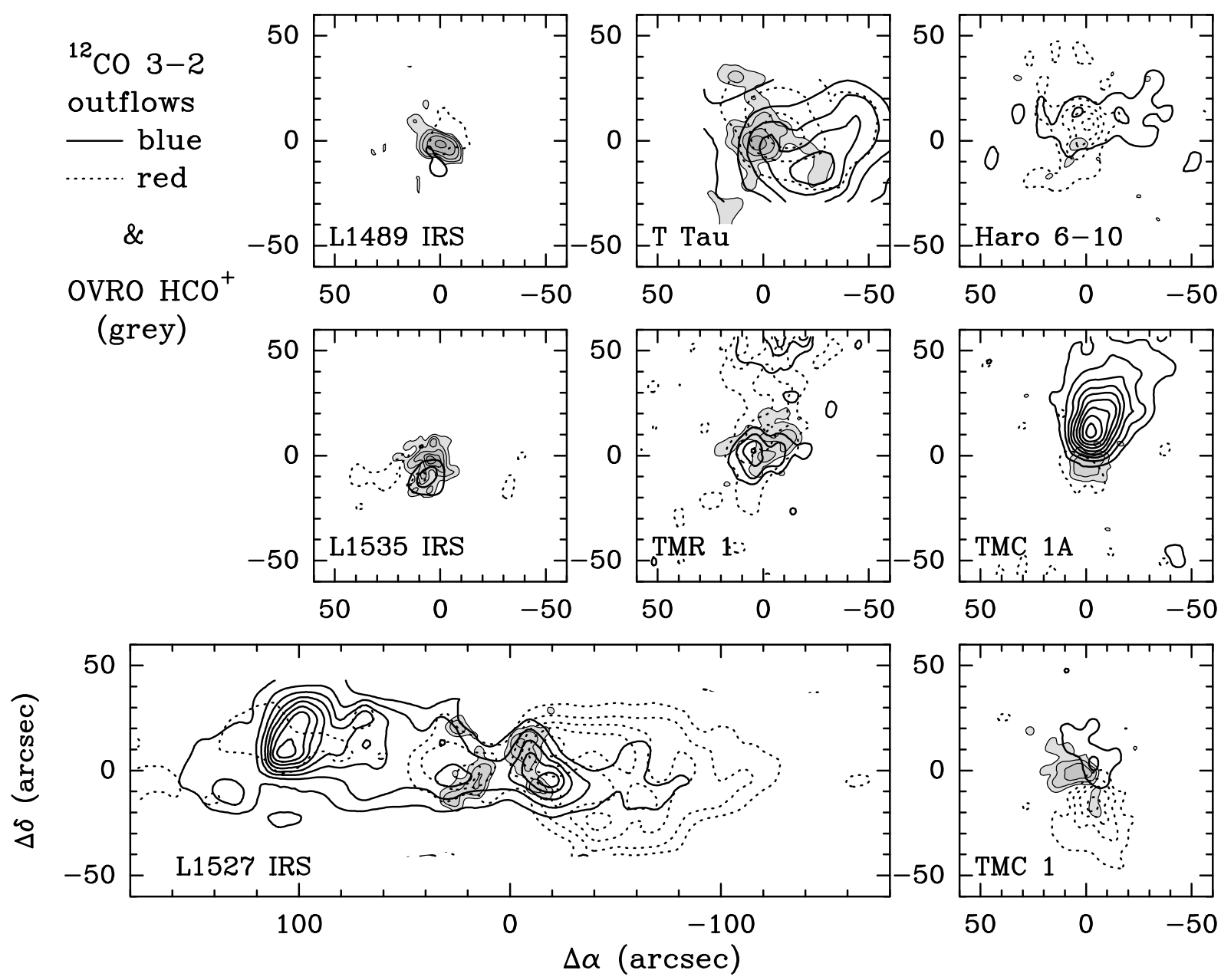

FIG. 9. - Overlay of the ${ }^{12} \mathrm{CO} 3-2$ maps from Fig. 6 (contours) on the integrated $\mathrm{HCO}^{+} 1-0$ images from Fig. 1 (grey scale). For L1489 IRS, the zero-moment image of $\mathrm{HCO}^{+}$has been plotted, which better brings out the low-level emission extending along the northern outflow lobe.

$\mathrm{HCO}^{+} 1-0$ and ${ }^{13} \mathrm{CO}$ redshifted by $0.5 \mathrm{~km} \mathrm{~s}^{-1}$ with respect to the systemic velocity. Van Langevelde et al. (1994a) tentatively ascribe the absorption to optically thick, warm, infalling gas. In the single-dish beam, the absorption is completely filled in by emission (Fig. 4).

\subsubsection{Haro 6-10 (GV Tau)}

In ${ }^{13} \mathrm{CO}$ emission, a small core is seen by OVRO toward this source, while only a marginal detection of $\mathrm{HCO}^{+}$is obtained. $\mathrm{C}^{18} \mathrm{O}$ is undetected. A molecular mass of only $0.0005-0.003 M_{\odot}$ is inferred from the ${ }^{13} \mathrm{CO}$ emission, consistent with the upper limit on the dust mass from Paper I. Single-dish $\mathrm{HCO}^{+} 1-0$ observations show extended emission lacking a central concentration; the $\mathrm{HCO}^{+} 4-3$ emission is also relatively diffuse. Combined with the unresolved $K^{\prime}$ emission (Fig. 10) and the ill-defined outflow structure (Fig. 6), we conclude that most of the envelope of this source has already disappeared. This is consistent with the large relative age inferred for the object in Paper I, as traced by the ratio of $\mathrm{HCO}^{+} 3-2$ intensity to $L_{\mathrm{bol}}$. This source is clearly more evolved than $\mathrm{T}$ Tau, although, like $\mathrm{T}$ Tau, it consists of a $\mathrm{T}$ Tauri star and a more embedded infrared companion. This supports the explanation offered by Koresko, Herbst, \& Leinert (1997) and Hogerheijde et al. (1997b), that infrared companions are being viewed at a special orbital phase or geometry of the binary system, but are otherwise at the same evolutionary stage as the primary object.

\subsubsection{L1551 IRS 5}

This source is one of the best-studied low-mass YSOs, not least because of its spectacular outflow. The outflow, which has a position angle of $45^{\circ}$ with the blue lobe extending to the southeast, has been mapped by Moriarty-Schieven \& Snell (1988) and is not presented here. Recent results of Looney, Mundy, \& Welch (1997) have shown that this object is a $50 \mathrm{AU}$ separation binary. Interferometric observations have been reported by Sargent et al. (1988), Rudolph (1992), and Ohashi et al. (1996a, 1996b). Consistent with these works, our $\mathrm{HCO}^{+},{ }^{13} \mathrm{CO}$, and $\mathrm{C}^{18} \mathrm{O}$ OVRO observations reveal a marginally resolved core at the source center that is slightly elongated perpendicular to the outflow in ${ }^{13} \mathrm{CO}$ and $\mathrm{C}^{18} \mathrm{O}$, as well as several $\mathrm{HCO}^{+}$ clumps scattered around the field of view. From the $K^{\prime}$ image (Fig. 10), it is seen that the ${ }^{13} \mathrm{CO}$ and $\mathrm{C}^{18} \mathrm{O}$ emission outlines the east side of the comma-shaped scattered-light feature, tracing the base of the outflow cavity (Fig. 1). The detailed anticorrelation of $\mathrm{HCO}^{+}$and scattered light suggests that the $\mathrm{HCO}^{+}$clumps correspond to condensations in the material surrounding the cavity. Comparison with the single-dish $\mathrm{HCO}^{+}$results (Figs. 3 and 4) shows that most of the large-scale emission is resolved out by OVRO. 


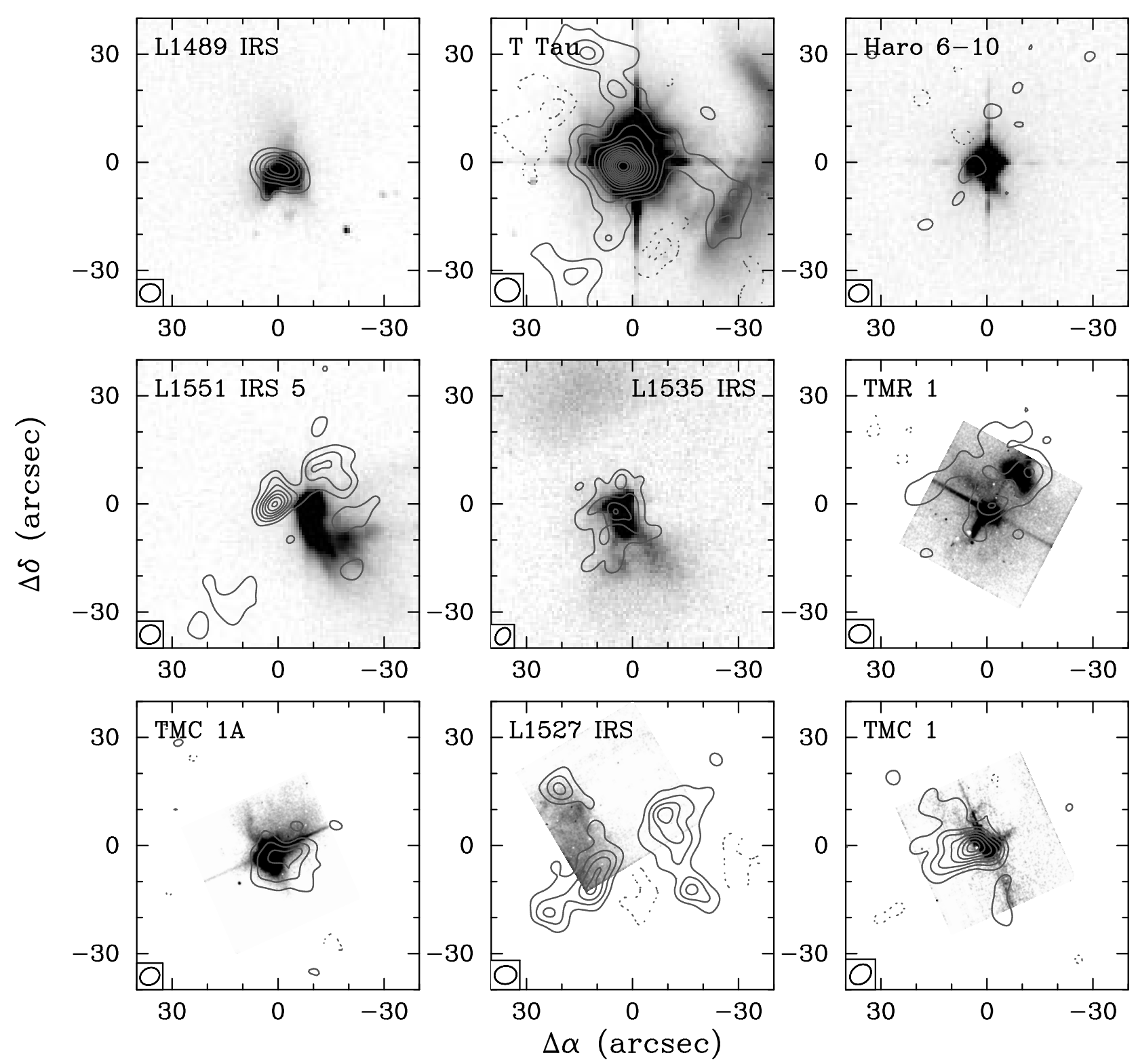

FIG. 10.- $K^{\prime}$ scattered-light images from Hodapp (1994) of L1489 IRS, T Tau, Haro 6-10, L1551 IRS 5, and L1535 IRS, with half-power wavelengths of 1.94 and $2.29 \mu \mathrm{m}$, and unpublished Keck telescope data of TMR 1, TMC 1A, L1527 IRS, and TMC 1, over 1.995-2.292 $\mu \mathrm{m}$ (grey scale), overlaid with the $\mathrm{HCO}^{+}$images from Fig. 1 (contours). The near-infrared and millimeter data have been aligned by eye with the stellar position for T Tau, Haro 6-10, TMC 1A, and TMC 1. The image of L1527 IRS was shifted to the same position as given by Tamura et al. (1991), while the remaining sources were aligned in such a way that a clear correspondence was found between features in the $\mathrm{K}^{\prime}$ and $\mathrm{HCO}^{+}$images.

The central, elongated $\mathrm{C}^{18} \mathrm{O}$ core contains $0.1 M_{\odot}$, or $40 \%$ of the single-dish dust mass. The position-velocity diagram obtained along the major axis suggests rotation around a $\sim 0.5 M_{\odot}$ object. This is uncomfortably low for a binary system, and much smaller than the $2.6 M_{\odot}$ found from $L_{\mathrm{bol}}$. The latter value may be overestimated, if L1551 IRS 5 is undergoing a FU Orionis outburst, as has been suggested by Mundt et al. (1985). In addition, the gas may not be on Keplerian orbits, as proposed by Saito et al. (1996) and Ohashi et al. (1996a), who model velocity structure of the $\mathrm{H}^{13} \mathrm{CO}^{+}$and ${ }^{13} \mathrm{CO}$ emission with disklike infall toward a $0.5-1.0 M_{\odot}$ object. The position-velocity diagram shows a tail of redshifted emission associated with the outflow. The $\mathrm{HCO}^{+}$and ${ }^{13} \mathrm{CO}$ spectra show deep selfabsorption close to $V_{0}$ due to optically thick, extended material.
The inferred systemic velocity of $6.7 \mathrm{~km} \mathrm{~s}^{-1}$ differs significantly from the value of $\sim 6.2 \mathrm{~km} \mathrm{~s}^{-1}$ found by Sargent et al. (1988) and Ohashi et al. (1996a). L1551 IRS 5 is the only source for which we find a difference in $V_{0}$ from published values. We believe that this difference is spurious, although careful examination of the observational settings did not reveal any discrepancies. The exact value of $V_{0}$ has no influence on any of our conclusions.

\subsubsection{L1535 IRS}

The integrated OVRO images of $\mathrm{HCO}^{+},{ }^{13} \mathrm{CO}$, and $\mathrm{C}^{18} \mathrm{O}$ emission from L1535 IRS (Fig. 1) reveal a core of $0.002 M_{\odot}$, coincident with the peak of the large-scale emission (Fig. 3). A similar mass of $0.0065 M_{\odot}$ is inferred by Ohashi et al. (1996b) from interferometer CS measurements and the $1.1 \mathrm{~mm}$ continuum upper limit of $0.01 M_{\odot}$ (Paper I). 

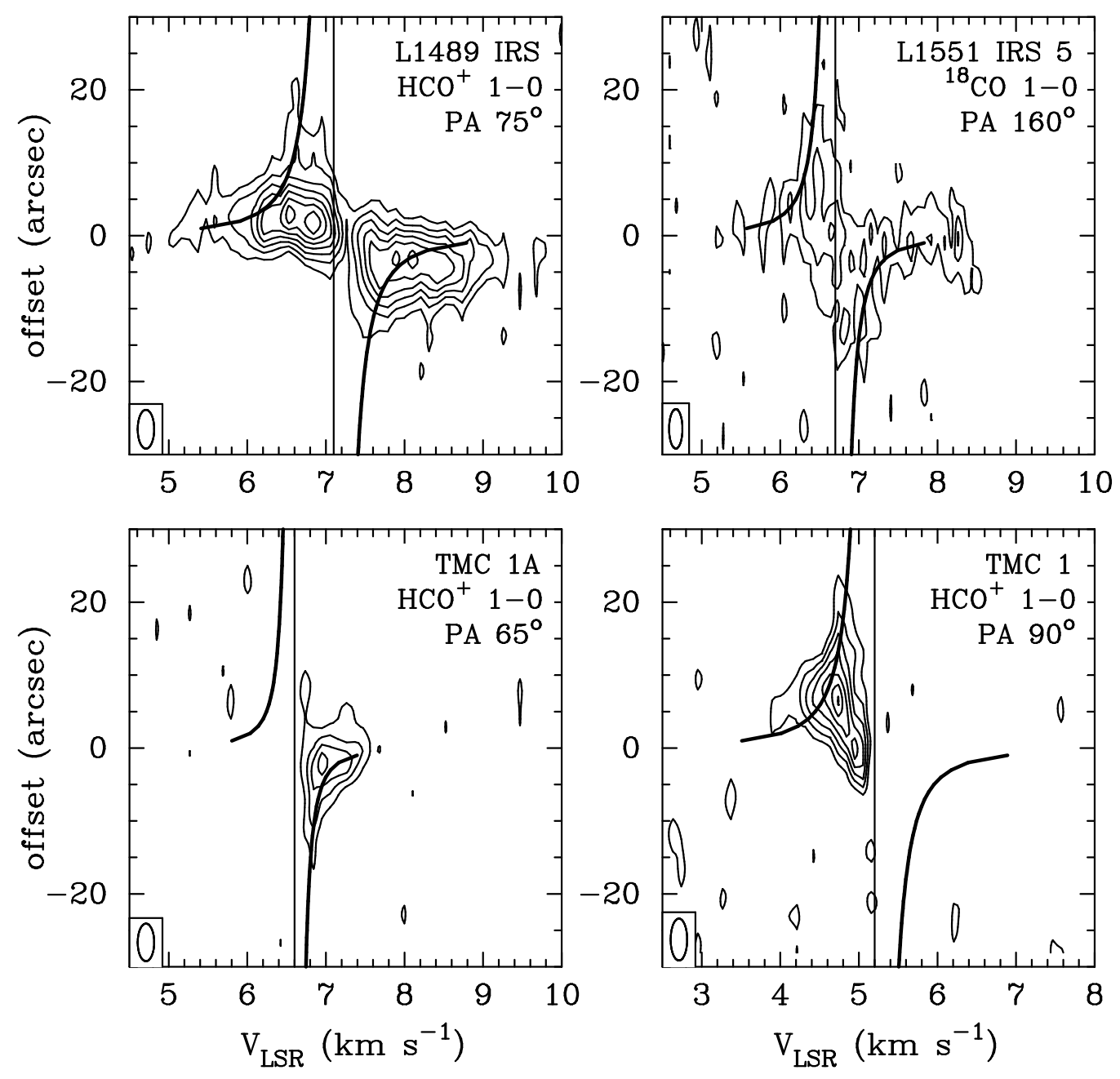

FIG. 11.-Position-velocity diagrams of $\mathrm{HCO}^{+}$and $\mathrm{C}^{18} \mathrm{O}$ along the major axes of the cores observed toward L1489 IRS, L1551 IRS 5, TMC 1A, and TMC 1. The systemic velocity of the objects is indicated by the vertical line. For comparison, Keplerian rotation is shown for inclination-corrected stellar masses of $0.9 M_{\odot}(\mathrm{L} 1489 \mathrm{IRS}), 0.5 M_{\odot}$ (L1551 IRS 5), $0.2 M_{\odot}$ (TMC 1A), and $0.8 M_{\odot}$ (TMC 1). Additional contributions from the outflows are likely for L1489 IRS, and for the redshifted tail of $\mathrm{C}^{18} \mathrm{O}$ toward L1551 IRS 5.

At $4.8 \mathrm{~km} \mathrm{~s}^{-1}$, the $\mathrm{C}^{18} \mathrm{O}$ emission is blueshifted by $0.7 \mathrm{~km}$ $\mathrm{s}^{-1}$ from the single-dish systemic velocity, possibly tracing velocity structure, like rotation close to the source. Although no obvious outflow structure is seen in ${ }^{12} \mathrm{CO} 3-2$ (Fig. 6), the $K^{\prime}$ observations show a clear bipolar reflection nebulosity, with a position angle of $10^{\circ}$. A close agreement is found between details in the $\mathrm{HCO}^{+}$and $K^{\prime}$ emission, for example the straight edge on the west side and the extensions to the south and along the northern edge. Approximately $30^{\prime \prime}$ north of the source, a secondary $K^{\prime}$ emission region is visible, the nature of which is not entirely clear. On the single-dish $\mathrm{HCO}^{+}$1-0 map of Figure 3, its location coincides with a depression in the $\mathrm{HCO}^{+}$emission. Possibly, the lower column density allows scattered light to escape. Alternatively, the dark lane separating the two $K^{\prime}$ emission regions could be interpreted as enhanced extinction. A similar feature is seen toward TMR 1 (see next section), and Whitney et al. (1997) show that it cannot be due to absorption, since it is bluer than the surrounding emission.

\subsection{6. $T M R 1$}

In the OVRO beam a $\sim 15^{\prime \prime}$ core is seen in $\mathrm{HCO}^{+}$and ${ }^{13} \mathrm{CO}$ toward this source, while only weak emission is observed in $\mathrm{C}^{18} \mathrm{O}$. A mass of $0.009 M_{\odot}$ is inferred for the core, similar to the dust mass of $0.007 M_{\odot}$ from Paper I and the $0.01 M_{\odot}$ inferred by Ohashi et al. (1996b) from CS interferometry. In $K^{\prime}$ (Terebey et al. 1990), bipolar nebulosity is seen to be coincident with the ${ }^{12} \mathrm{CO} 3-2$ outflow (Fig. 6), which is intersected by an absorption lane $\sim 15^{\prime \prime}$ to the north of the source. However, Whitney et al. (1997) show that this band is bluer than the surrounding emission, indicating that it cannot be due to enhanced extinction. Instead, it seems likely that the secondary emission feature is caused by scattering off a condensation in the surrounding cloud. In $\mathrm{HCO}^{+}$, and less clearly in ${ }^{13} \mathrm{CO}$, enhanced emission coincides with the north side of the $K^{\prime}$ feature, analogous to the association of $\mathrm{HCO}^{+}$with the reflection nebula NGC 1555 toward T Tau, supporting this interpretation (Fig. 10). The $\mathrm{HCO}^{+}$and ${ }^{13} \mathrm{CO}$ spectra consist of 2-3 sharp emission peaks, similar to the structure seen in the ${ }^{13} \mathrm{CO}$ single-dish spectrum (Fig. 5), which is probably caused by narrow and deep absorption features separating the peaks.

\subsubsection{TMC $1 A$}

In spite of the spectacular outflow driven by this object, the $\mathrm{HCO}^{+},{ }^{13} \mathrm{CO}$, and $\mathrm{C}^{18} \mathrm{O}$ emission observed by OVRO 
is confined to a $\sim 20^{\prime \prime}$ FWHM core around the source center. A mass of $0.004 M_{\odot}$ is inferred, or about $20 \%$ of the dust mass from Paper I. Extended $K^{\prime}$ emission can be seen to outline the base of the blue outflow lobe (Fig. 10). The velocity width of the $\mathrm{HCO}^{+}$emission is only $1 \mathrm{~km} \mathrm{~s}^{-1}$, corresponding to the red part of the single-dish spectrum, while the peak of the OVRO $\mathrm{HCO}^{+}$emission is offset by $\sim 3^{\prime \prime}$ to the west from the continuum position. When interpreted as rotation, the $\mathrm{HCO}^{+}$position-velocity diagram of Figure 11 indicates a stellar mass of $0.2 M_{\odot}$, after correcting for an inclination of $55^{\circ}$. This is in good agreement with the $0.3 M_{\odot}$ inferred from $L_{\text {bol }}$ (Table 1).

While the $\mathrm{HCO}^{+}$emission occurs close to the systemic velocity of $6.6 \mathrm{~km} \mathrm{~s}^{-1}$, the ${ }^{13} \mathrm{CO}$ and $\mathrm{C}^{18} \mathrm{O}$ emission is blueshifted by $2-3 \mathrm{~km} \mathrm{~s}^{-1}$, indicating their close association with the outflow. A similar trend is seen in the single-dish spectra of ${ }^{12} \mathrm{CO}$ and $\mathrm{HCO}^{+}$, where the ${ }^{12} \mathrm{CO}$ show prominent line wings of $15 \mathrm{~km} \mathrm{~s}^{-1}$, while the $\mathrm{HCO}^{+}$line extends over no more than a few $\mathrm{km} \mathrm{s}^{-1}$.

\subsubsection{L1527 IRS}

In this source, the association of the OVRO $\mathrm{HCO}^{+}$emission with the outflow is striking. Cross-shaped $\mathrm{HCO}^{+}$emission is seen to outline in detail the ${ }^{12} \mathrm{CO} 3-2$ outflow lobes (Fig. 9). The center of the $X$ is missing, because optically thick foreground emission is resolved out by OVRO. This is filled in by $\mathrm{H}^{13} \mathrm{CO}^{+}$, which shows a core elongated perpendicular to the outflow direction (Kitamura et al. 1997). The velocity extent of $3 \mathrm{~km} \mathrm{~s}^{-1}$ is similar to the single-dish line width, as is the overall shape of the line profile. Due to its lower resolution, the single-dish $\mathrm{HCO}^{+} 1-0$ map obtained with the IRAM $30 \mathrm{~m}$ shows no trace of the cross seen by OVRO, but only a core elongated in the direction of the outflow. In ${ }^{13} \mathrm{CO}$, a bow-tie-shaped core is detected, filling in the central region where $\mathrm{HCO}^{+}$emission is optically thick. No $\mathrm{C}^{18} \mathrm{O}$ emission is detected at the attained noise level. The total mass traced by ${ }^{13} \mathrm{CO}$ is $0.005-0.03 M_{\odot}$, depending on the ill-constrained opacity, and consistent with the $0.03 M_{\odot}$ inferred in Paper I from the dust continuum. Ohashi et al. (1997a) detected an elongated core in $\mathrm{C}^{18} \mathrm{O}$ with higher sensitivity Nobeyama Millimeter Array observations, and inferred a mass of $0.04 M_{\odot}$. Zhou, Evans, \& Wang (1996) find $0.7 M_{\odot}$, including zero-spacing information, while Fuller, Ladd, \& Hodapp (1996) infer 0.20$0.46 M_{\odot}$ from single-dish observations. The increased mass inferred over larger size scales, which are resolved out by the interferometer, indicates the presence of significant amounts of material around this deeply embedded source. $K^{\prime}$ emission is only detected $20^{\prime \prime}$ east of the source, coincident with the blue outflow lobe (Fig. 10; see also Tamura et al. 1991).

\subsection{9. $T M C 1$}

The $\mathrm{HCO}^{+},{ }^{13} \mathrm{CO}$, and $\mathrm{C}^{18} \mathrm{O}$ emission toward this source shows a wedge-shaped core offset to the east of the continuum position, with a mass of 0.007 , comparable to the dust mass of $0.005-0.016 M_{\odot}$ from Paper I. The velocity width of the OVRO and single-dish $\mathrm{HCO}^{+}$are small $(\sim 1$ $\mathrm{km} \mathrm{s}^{-1}$ ), with OVRO recovering $\sim 40 \%$ of the single-dish $\mathrm{HCO}^{+}$flux. Similar, slightly elongated cores perpendicular to the outflow are seen in the single-dish $\mathrm{HCO}^{+} 1-0,3-2$, and 4-3 maps (Fig. 3). These cores are interpreted as part of a rotating circumstellar envelope of $\sim 2500$ AU radius, of which the western, slightly redshifted half is obscured by optically thick foreground material. All $\mathrm{HCO}^{+}$emission is blueshifted with respect to the systemic velocity of $5.2 \mathrm{~km}$ $\mathrm{s}^{-1}$, indicating a central stellar mass of $\sim 0.8 M_{\odot}$ when interpreted as Keplerian rotation and assuming an inclination of $55^{\circ}$ (Fig. 11). This is much more than the $0.15 M_{\odot}$ inferred from $L_{\mathrm{bol}}$, which may be underestimated because of the deeply embedded nature of TMC 1 (cf. Yorke et al. 1995), as evidenced by the large line opacities.

Additional $\mathrm{HCO}^{+}$emission is seen coincident with the southern redshifted outflow lobe. The emission occurs at a $V_{\text {LSR }}$ of $4.9 \mathrm{~km} \mathrm{~s}^{-1}$, blueshifted by only $0.3 \mathrm{~km} \mathrm{~s}^{-1}$ from the systemic velocity, indicating that it traces material in the cavity wall rather than entrained in the outflow. Weak $K^{\prime}$ emission is seen to coincide with the base of the blue outflow lobe (Fig. 10).

\subsection{General Trends}

The interpretation of the OVRO molecular line data is complicated by the missing short-spacing fluxes, resulting in the absence of all extended emission, and by the often significant opacity in the $\mathrm{HCO}^{+}$and ${ }^{13} \mathrm{CO}$ lines. However, the observations can be explained in terms of the following components: a core of semimajor axis $\$ 1000$ AU around the star, which may be flattened or rotating, condensations scattered throughout the envelope, and material within the outflow or along the outflow cavity walls. Several of these components are also identified in millimeter line aperture synthesis observations of other embedded YSOs by, e.g., Langer, Velusamy, \& Xie (1996) for B5 IRS 1, Gueth et al. (1997) for L1157, and Hirano et al. (1997) for B1.

Cores surrounding the central star are most clearly seen in $\mathrm{HCO}^{+}$around L1489 IRS, and in $\mathrm{C}^{18} \mathrm{O}$ toward L1551 IRS 5. Toward TMC 1 only the western, blueshifted half of a rotating envelope appears to be present in $\mathrm{HCO}^{+}$. The other half may be obscured by optically thick foreground material. TMC 1A only shows the eastern, redshifted half, while for L1527 IRS this component is traced by $\mathrm{C}^{18} \mathrm{O} 1-0$ in the higher signal-to-noise ratio data of Ohashi et al. (1997a) and the $\mathrm{H}^{13} \mathrm{CO}^{+}$data of Kitamura et al. (1997). The masses of these cores are typically a few times $10^{-3} M_{\odot}$, but as high as $0.1 M_{\odot}$ for L1551 IRS 5, and amount to $20 \%$ $100 \%$ of the material sampled by the single-dish $1.1 \mathrm{~mm}$ continuum observations. The velocity gradients perpendicular to the outflow direction to L1489 IRS, L1551 IRS 5, TMC 1A, and TMC 1 are suggestive of rotation, but could also contain contributions from infalling or outflowing material.

Around T Tau, L1551 IRS 5, L1535 IRS, and TMR 1, the interferometer observations pick up condensations scattered throughout the envelopes in $\mathrm{HCO}^{+}$and ${ }^{13} \mathrm{CO}$. These may be inhomogeneities left over from the original cloud core, or that have grown during the collapse phase. Often, they correlate with bright or dark patches in the $K^{\prime}$ image, depending on their location in front of or behind the outflow cavity. For example, the reflection nebula NGC 1555 to the southwest of T Tau coincides with a peak in $\mathrm{HCO}^{+}$; the $\mathrm{HCO}^{+}$emission regions around L1551 IRS 5 seem to correspond to clumps of enhanced extinction, tracing out the comma-shaped $K^{\prime}$ emission; and the patches of scattered light around TMR 1 all correlate with features in $\mathrm{HCO}^{+}$emission.

A number of sources shows emission in the interferometer that is associated with, but not necessarily entrained in, the outflow. This is seen toward L1527 IRS, where the 
$\mathrm{HCO}^{+}$outlines the ${ }^{12} \mathrm{CO} 3-2$ in detail, but also toward L1551 IRS 5, where the ${ }^{13} \mathrm{CO}$ and $\mathrm{C}^{18} \mathrm{O}$ emission curves around the base of the southwest outflow, and toward TMC 1 , where $\mathrm{HCO}^{+}$emission coincides with the southern outflow lobe. All this emission is within $1-2 \mathrm{~km} \mathrm{~s}^{-1}$ of the systemic velocity, and is probably not entrained within the outflow. Instead, it traces material in the walls of the outflow cavity that is compressed or heated by the outflow. The amount of material incorporated in the bright cavity walls toward L1527 IRS is estimated at no more than a few percent of the total envelope mass. Although the $\mathrm{HCO}^{+}$ flux in the OVRO beam amounts to $30 \%-50 \%$ of the $30^{\prime \prime}$ single-dish value, the optical depth of $10-30$ obscures the inner envelope, which is recovered in $\mathrm{H}^{13} \mathrm{CO}^{+}$(Kitamura et al. 1997), and which contains most of the mass. The prominence of the cavity walls in $\mathrm{HCO}^{+}$is probably due to increased temperature or density, revealing the interaction of the outflow with the surrounding envelope material. Alternatively, the temperature in these walls may be increased through ultraviolet photons originating from the star-disk boundary layer, which scatter from the cavity (cf. Spaans et al. 1995). In this case, the role of the outflow is limited to clearing a low-opacity pathway for the heating photons.

Toward a few sources, red or blueshifted ${ }^{13} \mathrm{CO}$ and $\mathrm{C}^{18} \mathrm{O}$ emission is picked up, associated with material entrained in the outflow. The $\mathrm{C}^{18} \mathrm{O}$ position-velocity diagram of L1551 IRS 5 shows a high-velocity tail that may be associated with gas at the base of the outflow. Similar high-velocity tails may be present in the $\mathrm{HCO}^{+}$position-velocity diagram of L1489 IRS. The ${ }^{13} \mathrm{CO}$ and $\mathrm{C}^{18} \mathrm{O}$ emission toward TMC $1 \mathrm{~A}$ is entirely blueshifted by $2-3 \mathrm{~km} \mathrm{~s}^{-1}$. Interferometer observations of ${ }^{12} \mathrm{CO}$ lines of a number of YSOs clearly show the presence of material entrained within the outflow (e.g., B5 IRS 1, Langer, Velusamy, \& Xie 1996; B1, Hirano et al. 1997).

\section{SUMMARY}

The 700 AU scale structure of the envelopes around a sample of nine embedded, low-mass YSOs is investigated by millimeter aperture synthesis imaging of $\mathrm{HCO}^{+},{ }^{13} \mathrm{CO}$, and $\mathrm{C}^{18} \mathrm{O} 1-0$. These observations are complemented by singledish observations of ${ }^{12} \mathrm{CO} 6-5$ and $4-3,{ }^{13} \mathrm{CO} 6-5$ and 3-2, and $\mathrm{C}^{18} \mathrm{O} 3-2$ and $2-1$ to constrain line opacity and excitation conditions, and ${ }^{12} \mathrm{CO} 3-2$ maps with $15^{\prime \prime}$ resolution to illustrate the connection between envelopes and outflows. Our main conclusions can be summarized as follows:

1. Compact emission in $\mathrm{HCO}^{+},{ }^{13} \mathrm{CO}$, and $\mathrm{C}^{18} \mathrm{O}$ is clearly detected toward all sources, except for Haro 6-10, which lacks central condensation in its envelope. Molecular masses of $0.001-0.1 M_{\odot}$ are inferred and are found to be comparable to the masses traced by $1.1 \mathrm{~mm}$ single-dish continuum measurements. Together with the similarly sized regions traced in $\mathrm{HCO}^{+} 1-0$ by OVRO, and in $\mathrm{HCO}^{+} 3-2$ and $4-3$ by the JCMT, this firmly supports the conclusion of Paper I that the latter are a reliable probe of the inner envelopes.

2. The OVRO line observations can be understood in terms of three components: (1) A core of radius $\sim 1000 \mathrm{AU}$, with masses in good agreement with those inferred from 1 $\mathrm{mm}$ single-dish dust continuum measurements. This core may be flattened and rotating. (2) Condensations scattered throughout the core, possibly left over from the inhomoge- neous structure of the original cloud, and which may have grown during collapse. (3) Material within the outflow or along the cavity walls. A fourth component, an unresolved $\left(<3^{\prime \prime}\right)$ circumstellar disk, is detected by OVRO measurements of the 3.4 and $2.7 \mathrm{~mm}$ continuum emission (Paper I). Higher resolution imaging at subarsecond resolution with an array in which the short spacings are recovered is essential to further investigate the physical and chemical structure of these envelopes.

3. On the scales sampled by the OVRO measurements, the $\mathrm{HCO}^{+}$abundance may be increased by a factor of a few on average compared to the single-dish observations from Paper I. Part of this increase can be explained by contamination arising from extended cloud $\mathrm{C}^{18} \mathrm{O}$ emission in the single-dish beam, and the assumed excitation temperatures and opacities. Our results indicate that $\mathrm{HCO}^{+}$is much better suited to trace small-scale structure around YSOs than, e.g., CS (cf. Ohashi et al. 1996b). Over an area of $20^{\prime \prime} \times 20^{\prime \prime}$, an $\mathrm{HCO}^{+}$abundance of $4 \times 10^{-8}$ is obtained, with a spread of a factor of 3 between different sources. At least part of this spread is likely to be caused by opacity effects, which have only been approximately corrected for (see $\S 3$ ). Interferometer observations of $\mathrm{H}^{13} \mathrm{CO}^{+}$are required to obtain more accurate estimates of the abundance.

4. All objects are associated with outflow emission, but the ${ }^{12} \mathrm{CO} 3-2$ outflows of two-thirds of the sample are remarkably small at $<1^{\prime}$, as compared to L1551 IRS $5\left(10^{\prime}\right)$ and L1527 IRS ( $\left.3^{\prime}\right)$. The inclination- and opacity-corrected outflow momentum rate, $F_{\mathrm{CO}}$, and kinetic luminosity, $L_{\text {kin }}$, are in excellent agreement with previous studies of comparable samples of YSOs (CB92, Saraceno et al. 1996; Bontemps et al. 1996), and show a tight correlation with envelope mass. This relationship can be followed down to the scale of the circumstellar disk, supporting the interpretation that more massive and denser envelopes have a larger mass infall rate, and hence drive a more powerful outflow. Over the time span extending from deeply embedded class 0 sources to revealed T Tauri stars, age is expected to influence the outflow. However, within our sample of embedded objects of similar evolutionary state, the small difference in relative age is not correlated with outflow force.

5. The association of compact $\mathrm{HCO}^{+}$with the outflow cavity walls toward, e.g., L1527 IRS indicates that the outflows, in turn, are involved in shaping the envelopes. No more than a few percent of the total amount of envelope material is estimated to be incorporated in these cavity walls. It is not yet clear how important the outflows are in actually dispersing the YSO surroundings, or whether they merely provide a low-opacity pathway for heating radiation to reach into the envelope.

The authors wish to thank the staffs of the JCMT, CSO, IRAM $30 \mathrm{~m}$, and OVRO telescopes for their assistance. Remo Tilanus and Fred Baas are thanked for carrying out part of the JCMT observations. Jocelyn Keene kindly assisted in calibrating the CSO data. Lee Mundy is acknowledged for useful discussions. M. R. H. is indebted to the Caltech Divisions of Geological and Planetary Sciences and Mathematics, Physics and Astronomy, and the Owens Valley Radio Observatory for hospitality, and to the Netherlands Organization for Scientific Research (NWO) and the Leids Kerkhoven-Bosscha Fonds for travel 
support. Research in Astrochemistry in Leiden is supported by NWO/NFRA through grant 781-76-015. G. A. B. gratefully acknowledges support provided by NASA grants NAGW-2297 and NAGW-1955. H. J. v. L. acknowledges support for his research from the European Union under contract CHGECT920011. The referee is thanked for providing constructive comments that improved the manuscript.

\section{REFERENCES}

Bachiller, R. 1996, ARA\&A, 34, 111

Bontemps, S., André, Ph., Terebey, S., \& Cabrit, S. 1996, A\&A, 311, 858

Boss, A. P. 1993, ApJ, 410, 157

Cabrit, S., \& André, Ph. 1991, ApJ, 379, L25

Cabrit, S., \& Bertout, C. 1990, ApJ, 348, 530 .1992, A\&A, 261, 274 (CB92)

Chandler, C. J., Terebey, S., Barsony, M., Moore, T. J. T., \& Gautier, T. N. 1996, ApJ, 471, 308

Cohen, M., Emerson, J. P., \& Beichman, C. A. 1989, ApJ, 339, 455

Edwards, S., \& Snell, R. L. 1982, ApJ, 261, 151

Fiedler, R. A., \& Mouschovias, T. C. 1992, ApJ, 391, 199

1993, ApJ, 415, 680

Flower, D. R., \& Launay, J. M. 1985, MNRAS, 214, 271

Frerking, M. A., \& Langer, W. D. 1982, ApJ, 256, 523

Fuller, G. A., Ladd, E. F., \& Hodapp, K.-W. 1996, ApJ, 463, L97

Galli, D., \& Shu, F. H. 1993, ApJ, 417, 220

Gregersen, E. M., Evans, N. J., II, Zhou, S., \& Choi, M. 1997, ApJ, 484, 256

Gueth, F., Guilloteau, S., Dutrey, A., \& Bachiller, R. 1997, A\&A, in press

Hayashi, M., Hasegawa, T., Ohashi, N., \& Sunada, K. 1994, ApJ, 426, 234

Herbst, T. M., Koresko, C. D., \& Leinert, Ch. 1995, ApJ, 444, L93

Herbst, T. M., Robberto, M., \& Beckwith, S. V. W. 1997, AJ, 114, 744

Herbst, W., et al. 1986, ApJ, 310, L71

Hirano, N., Kameya, O., Mikami, H., Saito, S., Umemoto, T., \& Yamamoto, S. 1997, ApJ, 478, 631

Hodapp, K.-W. 1994, ApJS, 94, 615

Hogerheijde, M. R., de Geus, E. J., Spaans, M., van Langevelde, H. J., \& van Dishoeck, E. F. 1995, ApJ, 441, L93

Hogerheijde, M. R., van Dishoeck, E. F., Blake, G. A., \& van Langevelde, H. J. 1997a, ApJ, 489, 293 (Paper I)

Hogerheijde, M. R., van Langevelde, H. J., Mundy, L. G., Blake, G. A., \& van Dishoeck, E. F. 1997b, ApJ, 490, L99

Kenyon, S. J., \& Hartmann, L. 1995, ApJS, 101, 117

Kenyon, S. J., Whitney, B. A., Gomez, M., \& Hartmann, L. 1993, ApJ, 414, 773

Kitamura, Y., Kawabe, R., Yamashita, T., \& Hayashi, M. 1990, ApJ, 363, 180

Kitamura, Y., Saito, M., Kawabe, R., \& Sunada, K. 1997, in IAU Symp. 182, Herbig-Haro Flows and the Birth of Stars, ed. B. Reipurt \& C. Bertout (Dordrecht: Kluwer), 381

Königl, A., \& Ruden, S. P. 1993, in Protostars and Planets III, ed. E. H. Levy \& J. I. Lunine (Tucson: Univ. Arizona), 641

Koresko, C. D., Herbst, T. M., \& Leinert, Ch. 1997, ApJ, 480, 741

Langer, W. D., \& Penzias, A. A. 1990, ApJ, 357, 477

Langer, W. D., Velusamy, T., \& Xie, T. 1996, ApJ, 468, L41

Li, Z.-Y., \& Shu, F. H. 1996, ApJ, 472, 211

Looney, L. W., Mundy, L. G., \& Welch, W. J. 1997, ApJ, 484, L157

Lucas, P. W., \& Roche, P. F. 1997, MNRAS, 286, 895

Lucas, R., \& Liszt, H. 1996, A\&A, 307, 237

MacLeod, J., Lorne, A., Harris, A., Tacconi, L., \& Schuster, K. 1994, JCMT Newsletter, Sep/Oct, 46

Mardones, D., Myers, P. C., Tafalla, M., Wilner, D. J., Bachiller, R., \& Garay, G. 1997, ApJ, 489, 719

Millar, T. J., Bennett, A., Rawlings, J. M. C., Brown, P. D., \& Charnley, S. B. 1991, A\&AS, 87, 585

Minchin, N. R., Ward-Thompson, D., \& White, G. J. 1995, A\&A, 298, 894

Mizuno, A., Ohishi, T., Hayashi, M., Ohashi, N., Sunada, K., Hasegawa, T., \& Fukui, Y. 1994, Nature, 368, 719

Momose, M., Ohashi, N., Kawabe, R., Hayashi, M., \& Nakano, T. 1996, ApJ, 470, 1001
Moriarty-Schieven, G. H., \& Snell, R. L. 1988, ApJ, 332, 364

Moriarty-Schieven, G. H., Wannier, P. G., Mangum, J. G., Tamura, M., \& Olmsted, V. K. 1995, ApJ, 455, 190

Moriarty-Schieven, G. H., Wannier, P. G., Tamura, M., \& Keene, J. 1992, ApJ, 400, 260

Mundt, R., Stocke, J., Strom, S. E., Strom, K. M., \& Anderson, E. R. 1985, ApJ, 297, L41

Ohashi, N., Hayashi, M., Ho, P. T. P., \& Momose, M. 1997a, ApJ, 475, 211

Ohashi, N., Hayashi, M., Ho, P. T. P., Momose, M., \& Hirano, N. 1996a, ApJ, 466, 957

Ohashi, N., Hayashi, M., Ho, P. T. P., Momose, M., Tamura, M., Hirano, N., \& Sargent, A. I. 1997b, ApJ, 488, 317

Ohashi, N., Hayashi, M., Kawabe, R., \& Ishiguro, M. 1996b, ApJ, 466, 317

Padman, R., Bence, S. J., \& Richer, J. S. 1997, in IAU Symp. 182, HerbigHaro Flows and the Birth of Low-Mass Stars, ed. B. Reipurth \& C. Bertout (Dordrecht: Kluwer), 123

Palla, F., \& Stahler, S. W. 1993, ApJ, 418, 414

Raga, A., \& Cabrit, S. 1993, A\&A, 278, 267

Rudolph, A. L. 1992, ApJ, 397, L111

Saito, M., Kawabe, R., Kitamura, Y., \& Sunada, K. 1996, ApJ, 473, 464

Saraceno, P., André, Ph., Ceccarelli, C., Griffin, M., \& Molinari, S. 1996, A\&A, 309, 827

Sargent, A. I., Keene, J., Masson, C., \& Beckwith, S. 1988, ApJ, 333, 936

Schinke, R., Engel, V., Buck, U., Meyer, H., \& Diercksen, G. H. F. 1985, ApJ, 299, 939

Schuster, K.-F., Harris, A. I., Andersen, N., \& Russel, A. P. G. 1993, ApJ, 412, L67

Schuster, K.-F., Harris, A. I., \& Russell, A. P. G. 1997, A\&A, 321, 568

Scoville, N. Z., Carlstrom, J. E., Chandler, C. J., Phillips, J. A., Scott, S. L., Tilanus, R. P. J., \& Wang, Z. 1993, PASP, 105, 1482

Scoville, N. Z., Sargent, A. I., Sanders, D. B., \& Claussen, M. J. 1986, ApJ, 303,416

Shu, F. H. 1977, ApJ, 214, 488

Shu, F. H., Najita, J., Galli, D., Ostriker, E., \& Lizano, S. 1993, in Protostars and Planets III, ed. E. H. Levy \& J. I. Lunine (Tucson: Univ. Arizona Press), 3

Snell, R. L., Loren, R. B., \& Plambeck, R. L. 1980, ApJ, 239, L17

Spaans, M., Hogerheijde, M. R., Mundy, L. G., \& van Dishoeck, E. F. 1995, ApJ, 455, L167

Stahler, S. W. 1988, ApJ, 332, 804

Strom, K. M., Strom, S. E., Wenz, M., Wolff, S. C., \& Morgan, J. 1986, ApJS, 62, 39

Tamura, M., Gatley, I., Waller, W., \& Werner, M. W. 1991, ApJ, 374, L25

Tamura, M., Ohashi, N., Hirano, N., Itoh, Y., \& Moriarty-Schieven, G. H. 1996, AJ, 112, 2076

Terebey, S., Beichman, C. A., Gautier, N., \& Hester, J. J. 1990, ApJ, 362, 63

Terebey, S., Chandler, C. J., \& André, Ph. 1993, ApJ, 414, 759

Terebey, S., Shu, F. H., \& Cassen, P. 1984, ApJ, 286, 529

Terebey, S., Vogel, S. N., \& Myers, P. C. 1989, ApJ, 340, 472

van Langevelde, H. J., van Dishoeck, E. F., \& Blake, G. A. 1994a, ApJ, 425, L45

van Langevelde, H. J., van Dishoeck, E. F., van der Werf, P. P., \& Blake, G. A. 1994b, A\&A, 287, L25

Velusamy, T., Kuiper, T. B. H., \& Langer, W. D. 1995, ApJ, 451, L75

Whitney, B. A., Kenyon, S. J., \& Gómez, M. 1997, ApJ, 485, 703

Wilner, D. J., \& Welch, W. J. 1994, ApJ, 427, 898

Yorke, H. W., Bodenheimer, P., \& Laughlin, G. 1995, ApJ, 443, 199

Zhou, S., Evans, N. J., II, \& Wang, Y. 1996, ApJ, 466, 296

Note added in proof.-After this manuscript was accepted, more detailed modeling of the molecular line excitation and radiative transfer was carried out. This shows that the $\mathrm{HCO}^{+}$abundance as derived in $\S 3$ may be overestimated by a factor of a few because of the different levels of resolved-out, large-scale emission in $\mathrm{C}^{18} \mathrm{O}$ and $\mathrm{HCO}^{+}$, respectively. Therefore, our conclusion that $\mathrm{HCO}^{+}$may be enhanced on small scales needs further analysis. This does not affect any of our other conclusions, or the main bearing of the paper. 